\title{
A Survey of Underactuated Mechanical Systems
}

\author{
Yang Liu ${ }^{\mathrm{a}}$, Hongnian $\mathrm{Yu}^{\mathrm{b}}$ \\ ${ }^{a}$ Centre for Applied Dynamics Research, School of Engineering, King's College, University of Aberdeen, \\ UK, e-mail: y.liu@abdn.ac.uk \\ ${ }^{b}$ School of Design, Engineering and Computing, Bournemouth University, Bournemouth, UK, e-mail: \\ yuh@bournemouth.ac.uk
}

\begin{abstract}
An underactuated mechanical system (UMS) is the system that has fewer independent control actuators than degrees of freedom to be controlled. Control of UMS is considered as one of the most active fields of research due to its diverse engineering applications. This survey reviews UMS from its history to the state-of-the-art research on modelling, classification, control methods, and to some extent, provides some unique insights for bottleneck issues of control and future research directions.

Keywords: underactuated mechanical system, underactuation, dynamics, control, robotics
\end{abstract}

\section{Abbreviations}

UMS - Underactuated mechanical system

DOF - Degrees of freedom

TORA - Translational oscillator with rotational actuator

IWP - Inertia-wheel pendulum

PBC - Passivity-based control

IDA - Interconnection and damping assignment

VTOL - Vertical take off and landing

PFL - Partial feedback linearization

SMC - Sliding mode control

FC - Fuzzy control 


\section{Introduction}

An UMS is the system that has fewer independent control actuators than DOF to be controlled. This class of systems have been the subject of active scientific research fuelled by their broad applications in different disciplines. This survey aims to give a comprehensive but refined review of UMS from its history to the state-of-the-art research from the point of view of control, and focuses on its applications in robotics. To some extent, this paper provides some unique insights for classification of UMS, bottleneck issues of control, and future research directions.

Studies on UMS can trace back to two decades ago when control of nonholonomic mechanical systems were of great interest by scientists $[1,2]$, and they generated interesting control problems $[3,4]$ to which traditional control theory was not applicable. Examples of such systems include, but not limited to, mobile robot, spacecraft, underwater vehicle, surface vessel, helicopter, space robot, and underactuated manipulator. UMS has attracted more attention in 1990s, and they have been widely used in aerospace and robotics, e.g. $[5,6]$. Control of UMS is now one of the most active research fields in control community.

Robots, mechatronics, and hybrid machines are extensively employed in engineering to replace labour force for economic manufacture. Aircrafts, satellites, and space platforms are designed for exploration and military. Micro-robots, precise instruments and operational tools are developed to replace original medical tools for non-invasive diagnosis. Novel mechatronic systems are designed for necessary needs from industry and healthcare. As a result, the efforts of scientists and engineers led to the active research on nonlinear control theory, optimal control, adaptive control, and others. People are appreciating the substantial accomplishments that have been achieved [7, 8], and they are enjoying the benefits brought by machines. With this satisfaction, stimulated by their appetencies of exploring, researchers are eager to solve new issues by creating new systems and theories. Coming back to UMS, the research of UMS becomes crucial, and the following questions have arisen naturally: 
- Can we control UMS better using our current control techniques?

- Is there any novel UMS that can solve our problems in practice?

These two questions more or less motivate current research of UMS, and to some extent, they also imply that control of UMS is still a major open problem.

The remainder of this survey is organized as follows. In Section 3, modelling of UMS is reviewed, and some UMS examples are given. In Section 4, classifications of UMS are given from different points of view. In Section 5, control problems of UMS are discussed, and some popular control methods are reviewed. In Section 6, current challenges of UMS are discussed from both theoretical and practical points of view. Finally, concluding remarks and future research directions are drawn in Section 7.

\section{Modelling}

\subsection{Equations of motion}

Mathematical modelling of UMS is vital as it provides fundamental base for control. The equations of motion of UMS used in most literatures were obtained from the simple Lagrangian mechanical system [9] expressed as below

$$
\frac{d}{d t}\left(\frac{\partial L}{\partial \dot{q}}\right)-\frac{\partial L}{\partial q}=F(q) u
$$

where $L=T-V, T$ is the kinetic energy, $V$ is the potential energy of the system, $q \in \Re^{n}$ denotes the configuration vector, $u \in \Re^{m}$ is the actuator input vector, and $F(q) \in \Re^{n \times m}$ is a non-square matrix of external forces. In $[10,11]$, Eq. (1) is rewritten as

$$
\sum_{j} d_{k j}(q) \ddot{q}_{j}+\sum_{i, j} \Gamma_{i j}^{k}(q) \dot{q}_{i} \dot{q}_{j}+g_{k}(q)=p_{k}^{T} F(q) u
$$


where $k=1,2, \ldots, n, p_{k}$ is the $k^{t h}$ standard basis in $\Re^{n}, d_{k j}$ is an element of inertia matrix, $g_{k}(q)=\partial V(q) / \partial q_{k}$, and $\Gamma_{i j}^{k}(q)$ are called Christoffel symbols and is defined as

$$
\Gamma_{i j}^{k}(q)=\frac{1}{2}\left(\frac{\partial d_{k j}(q)}{\partial q_{i}}+\frac{\partial d_{k i}(q)}{\partial q_{j}}-\frac{\partial d_{i j}(q)}{\partial q_{k}}\right)
$$

In the vector form, Eq. (1) is written as

$$
D(q) \ddot{q}+C(q, \dot{q}) \dot{q}+G(q)=F(q) u
$$

where $D(q) \in \Re^{n \times n}$ is the inertia matrix which is a positive definite symmetric matrix, $c_{i j}=\sum_{k=1}^{n} \Gamma_{k j}^{i}(q) \dot{q}_{k}$ is an element of $C(q, \dot{q}), C(q, \dot{q}) \dot{q} \in \Re^{n}$ contains two types of terms involving $\dot{q}_{i} \dot{q}_{j}$ that are called Centrifugal terms $(i=j)$ and Coriolis terms $(i \neq j)$, and $G(q)$ is the gravity term. The simple Lagrangian mechanical system expressed by Eq. (4) can represent both fully-actuated mechanical system and UMS according to the column rank of $F(q)$. The simple Lagrangian mechanical system is called a fully-actuated mechanical system if $m=\operatorname{rank}(F)=n$, or is called an UMS if $m<n$.

Assuming $F(q)=\left[\begin{array}{ll}0 & I_{m}\end{array}\right]^{T}$, the configuration vector can be partitioned without loss of generality as $q=\left[\begin{array}{ll}q_{u} & q_{a}\end{array}\right] \in \Re^{n-m} \times \Re^{m}$, where $q_{u}$ and $q_{a}$ denote the unactuated and actuated configuration vectors, respectively. After partitioning the inertia matrix $D(q)$ in Eq. (4), the equations of motion of UMS can be reformed as

$$
\left[\begin{array}{c}
D_{u} \\
D_{a}
\end{array}\right]\left[\begin{array}{c}
\ddot{q}_{u} \\
\ddot{q}_{a}
\end{array}\right]+\left[\begin{array}{c}
C_{u}(q, \dot{q}) \\
C_{a}(q, \dot{q})
\end{array}\right]\left[\begin{array}{c}
\dot{q}_{u} \\
\dot{q}_{a}
\end{array}\right]+\left[\begin{array}{c}
G_{u}(q) \\
G_{a}(q)
\end{array}\right]=\left[\begin{array}{l}
0 \\
u
\end{array}\right],
$$

where $D_{u} \in \Re^{(n-m) \times n}, D_{a} \in \Re^{m \times n}, C_{u}(q, \dot{q}) \in \Re^{(n-m) \times n}, C_{a}(q, \dot{q}) \in \Re^{m \times n}, G_{u}(q) \in \Re^{n-m}$, and $G_{a}(q) \in \Re^{m}$. In $[12,13]$, the partitioned model is also written as

$$
\left[\begin{array}{cc}
D_{u u}(q) & D_{u a}(q) \\
D_{a u}(q) & D_{a a}(q)
\end{array}\right]\left[\begin{array}{c}
\ddot{q}_{u} \\
\ddot{q}_{a}
\end{array}\right]+\left[\begin{array}{l}
h_{u}(q, \dot{q}) \\
h_{a}(q, \dot{q})
\end{array}\right]=\left[\begin{array}{l}
0 \\
u
\end{array}\right]
$$


where the vectors $h_{u}(q, \dot{q}) \in \Re^{n-m}$ and $h_{a}(q, \dot{q}) \in \Re^{m}$ contain centripetal, Coriolis, and gravitational terms. In order to facilitate analysis and control of UMS, the following fundamental properties are obtained from Eq. (4):

- The inertial matrix $D(q)$ is symmetric, uniformly positive definite, and bounded above and below, i.e.

$$
0<\alpha_{m}(q) I_{n} \leq D(q) \leq \alpha_{M}(q) I_{n}<\infty
$$

where $I_{n}$ is the $n \times n$ identity matrix, $\alpha_{m}(q)$ and $\alpha_{M}(q)$ are scalar positive constants;

- The two matrices $D(q)$ and $C(q, \dot{q})$ are not independent. In particular, the Centrifugal and Coriolis terms $C(q, \dot{q}) \dot{q}$ is uniquely defined, but the matrix $C(q, \dot{q})$ is not. So, using a proper definition of $C(q, \dot{q}), \dot{D}(q)-2 C(q, \dot{q})$ is skew-symmetric such that

$$
X^{T}[\dot{D}(q)-2 C(q, \dot{q})] X=0,
$$

where $X^{T}$ is the transpose of $X \in \Re^{n}$ which is an arbitrary vector [14];

- Defining the total energy of the system $E(q, \dot{q})$, for conservative systems, gives

$$
E(q, \dot{q})=T(q, \dot{q})+V(q)=\frac{1}{2} \dot{q}^{T} D(q) \dot{q}+V(q),
$$

then the following formula is obtained

$$
\dot{E}(q, \dot{q})=\dot{q}^{T}\left[F(q) u-\frac{\partial P(\dot{q})}{\partial \dot{q}}\right]
$$

where $P(\dot{q})$ is the dissipation term of UMS.

\subsection{Examples of $U M S$}

This section presents several well-known examples of UMS, including the Acrobot, the Pendubot, the cart-pole system, the crane system, the rotating pendulum, the IWP, the 
beam-and-ball system, the magnetic suspension system, the TORA system, the VTOL aircraft, and the surface vessel. All the examples are chosen due to the fact that for analysis and control purposes, the complexity of their control design are of great interests by researchers. The equations of motion of these UMS examples are unified in a vector form by Eq. (4), and their unique matrices are summarized in Table 1.

\subsubsection{The Acrobot and the Pendubot}

The Acrobot [15] and the Pendubot [16] are two-link manipulators with a single actuator at elbow and shoulder as shown in Figure 1, respectively. Both manipulators graphically seem to be similar, and have the same equations of motion. The only difference is the input matrix $F(q)$, where $F=\left[\begin{array}{ll}0 & 1\end{array}\right]^{T}$ is for the Acrobot, and $F=\left[\begin{array}{ll}1 & 0\end{array}\right]^{T}$ is for the Pendubot. The control task for both systems is to stabilize the two-link manipulator to its upright equilibrium point $\left(q_{1}=\frac{\pi}{2}\right.$ and $\left.q_{2}=0\right)$ from any initial condition. One popular control approach is to use energy-based control to swing up the system from its stable downward position to unstable upright position, and switches to a linear controller for stabilization $[15,16,17,18,19]$. Similar approach has been adopted by Lai et al. [20] to give a comprehensive unified control strategy for both two-link manipulators. Recent works by Albahkali et al. [21] and Jafari et al. [22] have given a fresh idea on swing-up control of these two systems through an impulse-momentum approach, but without exception of using energy. An interesting work done by Yabuno et al. [23] investigated the reachable and stabilizable areas of the Pendubot, and proposed a control strategy using high-frequency excitation under the circumstance when the state feedback of the second link is not available.

\subsubsection{The cart-pole system and the crane system}

The cart-pole system shown in Figure 2 (a) is a benchmark underactuated system that has been widely used as a test bed for nonlinear control research. The control task is to swing up the pendulum from its stable downward equilibrium position $\left(q_{1}=0\right.$ and $\left.q_{2}=\pi\right)$ to upright unstable equilibrium position $\left(q_{2}=0\right)$ while keeps the cart at its original 


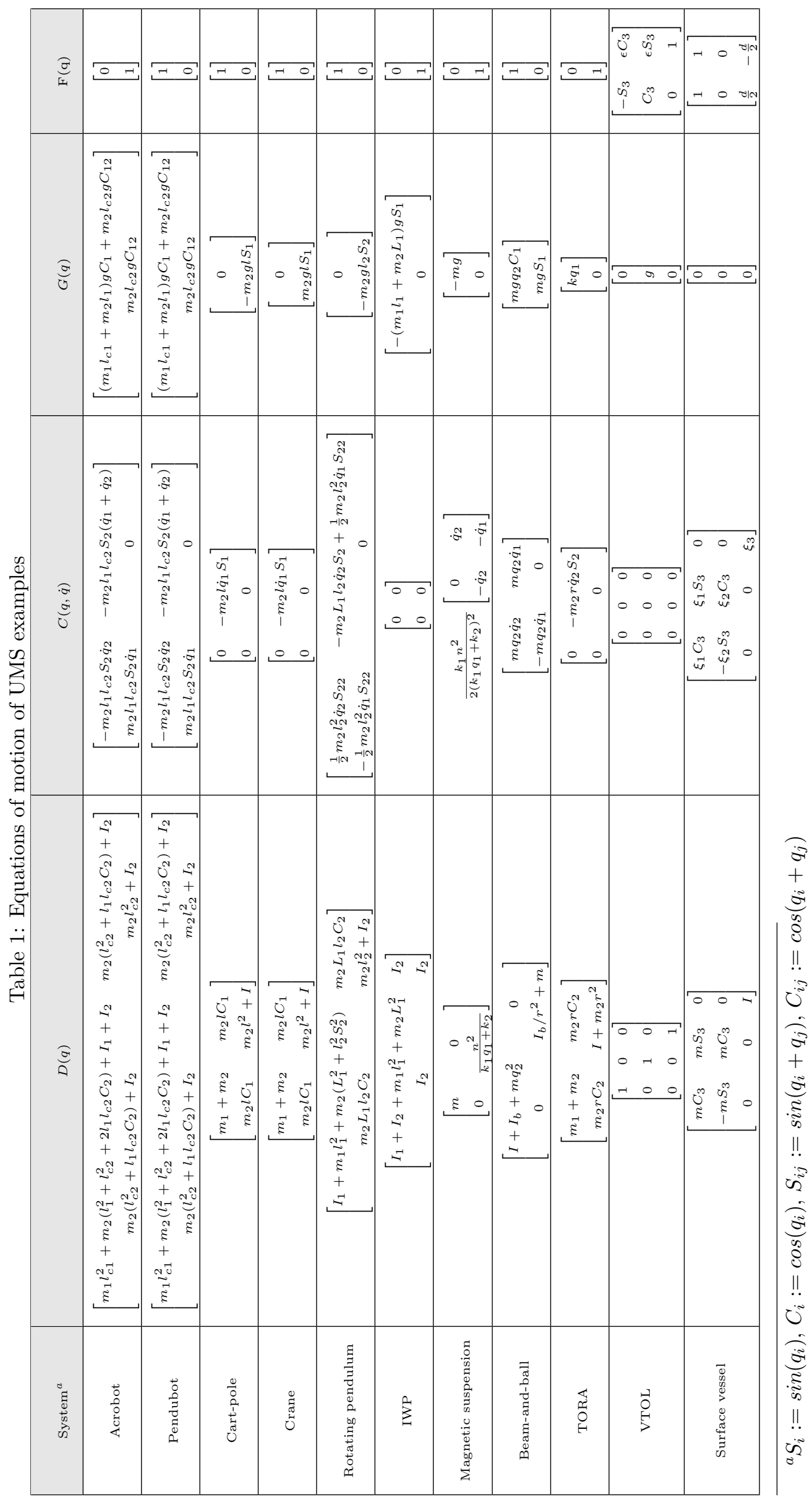


position $\left(q_{1}=0\right)$. The control works of the cart-pole system in the past two decades are huge from energy-based method to FC approach [24, 17, 25, 26, 27, 28], and its extended systems are massive, e.g. wheeled inverted pendulum [29, 30, 31], two-parallel-pendulum cart [32], two-serial-pendulum cart [33], 3-D pendulum cart [34], pendulum-driven cart $[35,36]$, and double-pendulum-driven cart [37]. Similar to the equations of motion of the cart-pole system, the crane system shown in Figure 2 (b) has a totally different control objective. The control task of the crane system is to reduce undesired payload swing by controlling the motion of the trolley. Some recent works on motion control of the crane system can be referred to $[38,39,40,41]$.

\subsubsection{The rotating pendulum}

The rotating pendulum (is also called Furuta pendulum) shown in Figure 3 is an inverted pendulum (Link 2) connecting to a rotating arm (Link 1). The control objective of the system is to swing up Link 2 from its stable downward position $\left(q_{2}=\pi\right)$ to the upright unstable position $\left(q_{2}=0\right)$ by rotating Link 1 , and balance it upright thereafter. To control the rotating pendulum, there is no exception of using energy-based control methods, e.g. $[42,43,44,45]$. An interesting work done by Freidovich et al. [46, 47] considered the problem of creating oscillations of the rotating pendulum rather than stabilizing it at the upright position.

\subsubsection{The inertia-wheel pendulum}

The IWP shown in Figure 4 consists of an unactuated pendulum with an actuated rotating uniform inertia wheel at its end. The IWP is a flat underactuated system with two-DOF and a single actuator acting on the wheel, and the control task is to swing up the pendulum by rotating the wheel, and stabilize it at its upright equilibrium position $\left(q_{1}=0\right)$ thereafter. The control methods for IWP include IDA-PBC [48], saturation technique [49], backstepping [50], and SMC [51]. 


\subsubsection{The magnetic suspension system}

The magnetic suspension system shown in Figure 5 is a typical nonlinear underactuated system that consists of an electromagnet, a suspended object, and the coil. The magnetic suspension system has been widely used in industrial applications, for examples, magnetic bearings, levitated vehicle, etc. Since its behavior is highly nonlinear and unstable, a feedback control system with high performance is always desirable. The control task is to move the suspended object from its initial position to a desired position under external disturbance. The control work on magnetic suspension system can be referred to $[52,53,54]$.

\subsubsection{The ball-and-beam system}

The ball-and-beam system [55] shown in Figure 6 contains a beam rotating on a vertical plane, an actuator mounted on the joint connecting the beam to the base, and a ball rolling freely along the beam. The control task is to move the ball from any initial position with any initial speed to a desired position on the beam by applying the torque to the beam. The well-established control works on the ball-and-beam system include IDAPBC [48], $\lambda$-method [56], a comparison of LQR, subspace stabilization, and combined error metric approaches [57], energy-based control [45], and Lyapunov-based method [58].

\subsubsection{The TORA system}

The TORA system shown in Figure 7 is a nonlinear benchmark example for the purpose of comparison and test of different control strategies. The system contains a translational oscillating platform and a rotational eccentric pendulum. The control task is to find a control law such that the horizontal displacement $q_{1}$ tends to zero in the presence of any external disturbance, and some control works can be referred to [59, 60, $61,62,63,64]$.

\subsubsection{The VTOL aircraft}

The simplified VTOL aircraft depicted in Figure 8 is an UMS with three-DOF and two control inputs. Under the assumption that $|\varepsilon|$ is relatively small, it is a slightly 
nonminimum phase system [65]. When $|\varepsilon| \gg 1$, it is a strongly non-minimum phase system which stabilization of such a system becomes difficult [66]. Most of the existing research on the VTOL aircraft has focused on set-point regulation $[67,68,69,70,71]$ and trajectory tracking $[72,73,74,75,76,77,78,79]$.

\subsubsection{The surface vessel}

The surface vessel shown in Figure 9 is a simplified model of underactuated surface ships with 3-DOF and two independent thrust inputs which have received considerable attention from the control community recently. The bulk of existing research has focused on global stabilization and tracking control [80,81, 82, 83, 84, 85, 86, 87], and with a special concern to its parametric uncertainties [88, 89, 90].

\section{Classification of UMS}

This section classifies UMS based on different system properties, and according to the redundancy and the fact that some of these properties have been less studied for practical applications, this paper only focuses on some particular ones that frequently appeared in the literatures.

\subsection{Classification by the reasons of underactuation}

It is known that the challenging issue of UMS is to control the system by using fewer independent actuators than DOF. In order to address this issue, it is necessary to understand the reasons that cause underactuation. The following reasons were summarized in [10] which can be used to classify UMSs:

- Dynamics of the system by nature, e.g. spacecraft, helicopter, underwater vehicle [91], wheeled mobile robot [92, 93];

- By design for reduction of the cost or some practical purposes, e.g. satellites with two thrusters [94, 95], flexible-link robot [96];

- Actuator failure, e.g. surface vessel, VTOL aircraft; 
- Imposed artificially to create complex low-order nonlinear systems for the purpose of gaining insight into controlling high-order UMS, e.g. the cart-pole system, the beam-and-ball system, the Acrobot, the Pendubot.

\subsection{Classification by system constraint}

Many UMSs are subject to their first-order or second-order constraints. The firstorder constraint is the constraint including generalized coordinate and velocity, and the second-order constraint is the constraint including generalized coordinate, velocity and acceleration. The differential constraint is holonomic if it is integrable, and is nonholonomic if it is non-integrable. An underactuated system is holonomic if its constraint is completely integrable, and is nonholonomic if its constraint is non-integrable or partial integrable [97]. Examples of holonomic and nonholonomic UMSs are given in Table 2.

Table 2: Classification by system constraint

\begin{tabular}{|c|c|c|}
\hline Class & Constraint & Examples \\
\hline $\begin{array}{c}\text { Holonomic } \\
\text { UMS }\end{array}$ & $\begin{array}{c}\text { Completely } \\
\text { integrable }\end{array}$ & $\begin{array}{c}\text { Horizontal pendulum [98], two-link } \\
\text { planar manipulator [97], pendulum- } \\
\text { driven cart }\end{array}$ \\
\hline $\begin{array}{c}\text { Nonholonomic } \\
\text { UMS }\end{array}$ & Partial integrable & Wheeled mobile robot, \\
or non-integrable & surface vessel, Acrobot, Pendubot \\
\hline
\end{tabular}

\subsection{Classification by configuration characteristics}

UMSs can be classified by the configuration characteristics [10] as follows: (a) actuated shape variables, (b) non-interacting inputs, (c) integrable normalized momentums, and (d) extra required conditions (details can be referred to [4]). The descriptions and examples of different classes of UMSs are given in Table 3.

\subsection{Classification by control problems}

The control problems of underactuated manipulators have been discussed in [99] which can be extended to control of UMS as below: 
Table 3: Classification by configuration characteristics [10]

\begin{tabular}{|c|c|c|c|c|c|}
\hline Class & $(\mathrm{a})$ & $(\mathrm{b})$ & $(\mathrm{c})$ & $(\mathrm{d})$ & Examples \\
\hline I & Yes $^{a}$ & Yes & Yes & No & Acrobot, IWP, TORA \\
\hline IIa & No & Yes & Yes & No & Rotating pendulum, Pendubot, beam-and-ball \\
\hline IIb & No & Yes & Yes & Yes & Flexible-link robot \\
\hline III & No & Yes & Yes & Yes & Cart-pole system \\
\hline IVa & Yes & No & Yes & No & Controlled VTOL Aircraft \\
\hline IVb & Yes & No & Yes & Yes & Controlled VTOL Aircraft \\
\hline V & Yes & No & Yes & Yes & VTOL aircraft, helicopter \\
\hline VIa & Yes & Yes & No & No & Three-link pendulum ${ }^{c}$ \\
\hline VIb & Yes & Yes & No & Yes & Three-link pendulum \\
\hline VIIa & No & Yes & No & No & Three-link pendulum \\
\hline VIIb & No & Yes & No & Yes & Three-link pendulum \\
\hline VIII & No & Yes & No & Yes & 3D cart-pole system \\
\hline
\end{tabular}

\footnotetext{
$a_{\text {a }}$ "Yes" or a "No" means the corresponding property holds or does not hold, respectively

${ }^{b}$ a controlled VTOL aircraft means the dynamics of the VTOL (in 3-DOF with two control inputs) in closed loop with a feedback $u_{1}=u_{1}\left(q_{1}, q_{2}, \dot{q}_{1}, \dot{q}_{2}\right)$ and a single remaining control $u_{2}$.

${ }^{c}$ the three-link pendulum is a 3 -DOF system with two control inputs, and the actuation configuration may lead the system to different classes.
}

Table 4: Classification by control problems

\begin{tabular}{|c|c|c|}
\hline Class & Control problems & Examples \\
\hline Car-like & $\begin{array}{c}\text { Trajectory planning } \\
\text { and tracking }\end{array}$ & $\begin{array}{c}\text { Wheeled mobile robot, surface vessel, } \\
\text { VTOL aircraft, helicopter, biped robot }\end{array}$ \\
\hline Pendulum-like & Set-point regulation & Cart-pole, Acrobot, Pendubot, \\
& & IWP \\
\hline
\end{tabular}


- Trajectory planning: given an initial configuration $q_{0}$ and a final desired configuration $q_{d}$, find a dynamically feasible trajectory that joins $q_{0}$ and $q_{d}$;

- Trajectory tracking: given a dynamically feasible trajectory $q_{d}(t)$, find a feedback control law that stabilizes the tracking error $e(t)=q_{d}(t)-q(t)$ to zero asymptotically;

- Set-point regulation: given a desired configuration $q_{d}$, find a feedback control law that stabilizes the equilibrium state $q=q_{d}$ and $\dot{q}=0$.

Based on the control problems above, UMSs can be classified into two categories as given in Table 4.

\section{Control of UMS}

Control of UMS aims to find a feedback control law that stabilizes the system in the presence of various uncertainties and external disturbances, and such a wide research field can be partitioned into two main categories: set-point regulation and trajectory tracking. The reason behind the complexity of this field is that the number of actuators is less than the degrees of freedom to be controlled, and many traditional nonlinear control methods are not directly applicable. In recent years, many control methods based on feedback linearization, energy, backstepping, sliding mode, and fuzzy logic have been developed for stabilization of UMS. This section provides a view to highlight some of these popular control methods.

\subsection{Partial feedback linearization}

A useful technique for control of UMS is the so-called PFL [24], which is a method providing a natural global change of coordinates that transforms the system into a strict feedback form, and the control method can be easily applied to the new form of the system. There are two PFL techniques presented as below. 


\subsubsection{Collocated PFL [17]}

Consider the actuated configuration vector $q_{a}$ in Eq. (6), there exists a global invertible change of control in the form

$$
u=\alpha_{1}(q) \tau+\beta_{1}(q, \dot{q})
$$

that partially linearizes Eq. (6) as follows

$$
\begin{aligned}
& \dot{q}_{u}=p_{u} \\
& \dot{p}_{u}=f_{0}(q, p)+g_{0}(q) \tau \\
& \dot{q}_{a}=p_{a} \\
& \dot{p}_{a}=\tau
\end{aligned}
$$

over the set

$$
U_{1}=\left\{q \in \Re^{n} \mid \operatorname{det}\left(D_{u u}(q)\right) \neq 0\right\},
$$

where $q=\left[\begin{array}{ll}q_{u} & q_{a}\end{array}\right]^{T}, \alpha_{1}(q)$ is an $\mathrm{m} \times \mathrm{m}$ positive definite symmetric matrix, and

$$
\begin{aligned}
f_{0}(q, p) & =-D_{u u}^{-1}(q) h_{u}(q, \dot{q}), \\
g_{0}(q) & =-D_{u u}^{-1}(q) D_{u a}(q) .
\end{aligned}
$$

Collocated PFL shows a procedure of linearization of actuated configuration variables which globally transforms all underactuated systems in the form of Eq. (6) to a fullyactuated form where the new control input appears in the dynamics of both subsystems. Olfati-Saber [10] further introduced a global change of coordinates that can decouple these two subsystems but left the linear subsystem invariant. The method has been extensively applied to control of the Acrobot [15], the three-link pendulum [17], the cart-pole system [28], the double pendulum cart [33], the pendulum-driven cart [36], and the capsule system [100]. 


\subsubsection{Non-Collocated PFL [10]}

Consider the following UMS

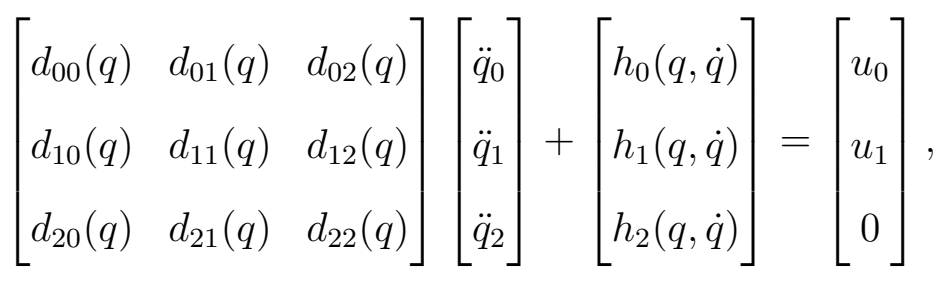

where $q=\left[\begin{array}{lll}q_{0} & q_{1} & q_{2}\end{array}\right]^{T}$. Then there exists a change of control in the form

$$
u=\alpha_{1}(q) \tau+\beta_{1}(q, \dot{q})
$$

where $u=\left[\begin{array}{lll}u_{0} & u_{1} & 0\end{array}\right]^{T}, \tau=\left[\begin{array}{lll}\eta & \sigma & 0\end{array}\right]^{T}$ and

$$
\sigma=\alpha_{0}(q) \eta+\alpha_{2}(q) \varphi+\beta_{2}(q, \dot{q})
$$

that partially linearizes Eq. (12) as below

$$
\begin{aligned}
& \dot{q}_{0}=p_{0} \\
& \dot{p}_{0}=\eta \\
& \dot{q}_{1}=p_{1} \\
& \dot{p}_{1}=f_{0}(q, p)+g_{0}(q) \eta+g_{2}(q) \varphi \\
& \dot{q}_{2}=p_{2} \\
& \dot{p}_{2}=\varphi
\end{aligned}
$$

over the set

$$
U=\left\{q \in \Re \Re^{n} \mid \operatorname{det}\left(d_{21}(q)\right) \neq 0\right\}
$$


where

$$
\begin{gathered}
f_{0}(q, p)=-d_{21}^{-1}(q) h_{2}(q, p), \\
g_{0}(q)=-d_{21}^{-1}(q) d_{20}(q), \\
g_{2}(q)=-d_{21}^{-1}(q) d_{22}(q)
\end{gathered}
$$

and $\varphi$ is the new control input.

Non-collocated PFL presents a procedure of linearization of unactuated configuration variables, and also holds for a restricted class of underactuated systems under the case that the number of unactuated configuration variables is less than or equal to the number of control inputs. Olfati-Saber [101] studied non-collocated PFL for underactuated systems with symmetry property, and transformed the non-triangular quadratic normal form into a cascade nonlinear system in a strict feedforward form. This method has been applied to control of the Pendubot [102], the rotating pendulum [101], the flexible one-link robot [103], and the surface vessel [104, 105]. The advantage of collocated and non-collocated PFL is a conceptual and structural simplification of control problem, i.e. they are always utilized as an initial simplifying step for reduction and control of UMS.

\subsubsection{A remark on flat UMS}

A flat UMS is the system that has the constant inertia matrix, $D(q)$ in Eq. (4), and $C(q, \dot{q})=0$ due to the vanishing Christoffel symbols in Eq. (3). The flat UMS has limited controllability as its gravity term of unactuated part, $G_{u}(q) \neq 0$ in Eq. (6). The IWP and the VTOL aircraft are the systems of this class, and their specific global change of coordinates $[10]$ is given as below.

Consider a flat UMS with configuration vector $q=\left(q_{x}, q_{s}\right)$ and a force matrix that is independent of $q_{x}$. Assume the potential energy of the system is independent of $q_{s}$ (i.e. 
$\left.V(q)=V\left(q_{x}\right)\right)$. The equations of motion for this system are written as

$$
\left[\begin{array}{cc}
m_{x x} & 0 \\
0 & m_{s s}
\end{array}\right]\left[\begin{array}{l}
\ddot{q}_{x} \\
\ddot{q}_{s}
\end{array}\right]+\left[\begin{array}{c}
-g_{x}\left(q_{x}\right) \\
0
\end{array}\right]=\left[\begin{array}{cc}
F_{r}\left(q_{s}\right) & F_{x}\left(q_{s}\right) \\
0 & F_{s}\left(q_{s}\right)
\end{array}\right]\left[\begin{array}{c}
\tau_{r} \\
\tau
\end{array}\right]
$$

where $g_{x}\left(q_{x}\right)=-\nabla_{q_{x}} V\left(q_{x}\right), \tau_{r} \in \Re, F_{r}\left(q_{s}\right): \Re^{m} \rightarrow \Re^{n-m}$ is a unit vector that is over a unit ball in $\Re^{n-m}, \tau \in \Re^{m}, m_{x x}$ and $m_{s s}$ are constant, and $F_{s}\left(q_{s}\right)$ is a $m \times m$ invertible matrix.

Let

$$
\tau=F_{s}\left(q_{s}\right)^{-1} m_{s s} u
$$

be the partially linearizing change of control. Assume all the elements of

$$
\omega=m_{x x}^{-1} F_{x}\left(q_{s}\right) F_{s}^{-1}\left(q_{s}\right) m_{s s} \cdot d q_{s}
$$

are exact one-forms and let $\omega=d \gamma\left(q_{s}\right)$. Then the following global change of coordinates

$$
\begin{aligned}
& q_{r}=q_{x}-\gamma\left(q_{s}\right) \\
& p_{r}=m_{x x} p_{x}-F_{x}\left(q_{s}\right) F_{s}^{-1}\left(q_{s}\right) m_{s s} p_{s}
\end{aligned}
$$

with $\left(p_{x}, p_{s}\right)=\left(\dot{q}_{x}, \dot{q}_{s}\right)$ transforms Eq. (13) into

$$
\begin{aligned}
& \dot{q}_{r}=m_{r}^{-1} p_{r} \\
& \dot{p}_{r}=g_{r}\left(q_{r}+\gamma\left(q_{s}\right)\right)-p_{s}^{T} \pi_{F}\left(q_{s}\right) p_{s}+F_{r}\left(q_{s}\right) \tau_{r} \\
& \dot{q}_{s}=p_{s} \\
& \dot{p}_{s}=u
\end{aligned}
$$

where $\pi_{F}\left(q_{s}\right)$ is a cubic matrix satisfying

$$
\frac{d}{d t}\left(F_{x}\left(q_{s}\right) F_{s}^{-1}\left(q_{s}\right)\right) m_{s s} p_{s}=p_{s}^{T} \pi_{F}\left(q_{s}\right) p_{s}
$$




\subsection{Energy-based methods}

\subsubsection{An introduction to passivity}

Considering the derivative of the total energy in Eq. (10) gives

$$
\dot{E}(q, \dot{q})=\dot{q}^{T}\left[F(q) u-\frac{\partial P(\dot{q})}{\partial \dot{q}}\right] \leq \dot{q}^{T} \cdot F(q) u
$$

which implies that the system is passive with respect to the input $u$ and output $\dot{q}$. The passivity is a vital character of UMS since a passive UMS has a stable origin and a feedback control law always exists for $\dot{E}(q, \dot{q}) \leq 0$.

The control methods based on the passivity of UMS are referred to passivity/energybased control. The main idea of these methods is to regulate the total energy of the system to the equivalent value of a desired equilibrium state by using Eq. (14). PBC is mostly used for set-up regulation of UMS, such as two-link manipulators [20], cart-pole [106], two-serial-pendulum cart [33], two-parallel-pendulum cart [32], rotating pendulum [47], TORA [107], and biped robot [108]. The main drawback of passivity/energy-based control is its narrow range of applications as no real-life applications in robotics and aerospace engineering have been found so far.

There are many energy-based control methods, such as $L_{2}$-gain method $[109,110]$ and direct Lyapunov method $[111,112]$, but the following review will focus on backstepping, IDA-PBC, and controlled Lagrangian methods according to their popularity.

\subsubsection{Backstepping}

One limitation of PBC is that it is only applicable to the systems with relative degree less than two. To overcome this limitation, a technique called backstepping is proposed to transform the system into a new recursive form which PBC can be easily applied to. The principle of backstepping [113] as depicted in Figure 10 is to design a sequence of "virtual" systems $S_{i}(i=1,2, \ldots, p)$ of relative degree one, finishing with the actual system as the last member of the sequence. For each "virtual" system $S_{i}$, the relative degree is reduced to one by selecting an available signal $\alpha_{i-1}$ as a "virtual" input and then achieving 
passivity with respect to a "virtual" output $\tau_{i}$. Finally, the actual control law $u=\alpha_{p}$ is obtained.

The backstepping technique is effective for global stabilization of the UMS with lowDOF. In recent years, it has been extensively applied to control surface vessel $[114,84$, $115,87,116,117]$ and VTOL aircraft $[66,76,118,119]$. However, when the DOF of UMS increases, the procedure of backstepping becomes very complicated, and implementation of such a control design in practical applications is unrealistic.

\subsubsection{IDA-PBC and controlled Lagrangian methods}

The Hamilton equation and the Euler-lagrange equation are two traditional ways to describe the behaviour of dynamic systems, and their systems are named as the Hamilton system and the Euler-lagrange system, respectively. There are also two mainly PBC methods for both UMSs: IDA-PBC [48, 120, 121] and controlled Lagrangian [122, 123]. As it is known, the equilibrium state of a Hamilton/Euler-lagrange system is related to a critical state of the Hamiltonian/Lagrangian. Both passivity-based methods consist of two stages: (1) shape the Hamiltonian/Lagrangian to a desired form with suitable equilibrium states and structural features by using control inputs, and then (2) inject damping into

the system to ensure the passivity of the system. A main difference between these two methods is that the desired Lagrangian in the controlled Lagrangian method is obtained by modifying only the generalized inertia matrix and the potential energy function, while IDA-PBC also considers the possibility of changing the interconnection matrix [48].

\subsection{Sliding mode control}

Uncertainty is a common yet intractable issue for the control of UMS. For any control design, there are typical discrepancies between a practical system and its theoretical model due to unmodelled dynamics, parameter uncertainty, and external disturbance. Adaptability and robustness to these factors has been an attractive research topic of control engineering in the past few decades. Robust control [124] and adaptive control [125] are two main techniques for uncertainty compensation, where the former is designed to ensure 
the system to be insensitive to all uncertainties by using a fixed control structure, while the later utilizes on-line identification of which either system parameter or controller parameter. In the earlier times, robust and adaptive control of the UMSs with uncertainties were considered by numbers of researchers. For instance, $\mathrm{Gu}[126]$ proposed a normal-form augmentation approach for recovering the parameter-linearity of the system, and then applied a direct adaptive control scheme to stabilize the UMS with parameter uncertainty. Shin [127] proposed a robust adaptive control method for underactuated manipulators with free-flying passive joints. Su and Stepanenko [128] studied a model-based adaptive variable structure control scheme for set-point regulation of underactuated manipulators. Behal et al. [80] designed a continuous time-varying adaptive tracking controller for a surface vessel in the presence of uncertainties associated with hydrodynamic damping coefficients. Do et al. [129] developed a nonlinear robust adaptive control strategy to control a surface vessel in the presence of environmental disturbances. However, due to the nature of these two control structures, robust control is only suitable for dealing with small uncertainty [130], while adaptive control is suitable for a wide range of parameter variation, but is sensitive to unstructured uncertainty.

To solve these limitations, a versatile variable-structure controller called SMC has received considerable devotion from researchers in recent years. SMC is insensitive to parameter variation and external disturbance, since the behaviour on the sliding mode depends only on the switching surface and is independent of the structural properties of the system. The basic idea of SMC is to alter the dynamics of the system by applying a discontinuous control input that forces the system to "slide" along a predefined state surface, and the system produces a desired behaviour by restricting its state to this surface. A schematics of SMC is shown in Figure 11, and its mathematical description is briefly given as follow. 
Consider a second-order system in cascade normal form

$$
\begin{aligned}
& \dot{x}=y, \\
& \dot{y}=f(x, y, t)+B(x, t) u(t),
\end{aligned}
$$

where $(x, y)$ is the system state, $f(x, y, t)$ and $B(x, t)$ are generally nonlinear functions, and $u(t)$ is the control input. Define a positive definite Lyapunov function

$$
V(t)=\frac{1}{2} s^{2}
$$

where $s(t)$ is a time varying surface. A SMC exists in the form such that

$$
u=u_{e q}+u_{s w}
$$

satisfying

$$
\dot{V}=s \cdot \dot{s} \leq 0
$$

where $u_{e q}$ is the equivalent control for maintaining the system state along the sliding surface $(s=0)$, and $u_{s w}$ is the switching control for driving the system states toward to the sliding surface.

SMC has succeeded in the applications of control of underactuated satellite [131], surface vessel [85, 132], helicopter [133], wheeled inverted pendulum [31], ball-and-beam system [134], underactuated fuel cell system [135], TORA [136], underactuated biped robot [137], and overhead crane [138]. Meanwhile, many researchers also devoted themselves to design universal SMC for the UMSs that have the same dynamic characteristics, e.g. $[139,98,140,71,141,142,143,144]$. Although SMC has grown exponentially in the past two decades, its applications on control of UMS is only mature at the level of simulation. Therefore, it is worth noting that "the real test for the sliding mode research community in the near future will be the willingness of control engineers to experiment 
with these SMC design approaches in their professional practice [145]."

\subsection{Fuzzy control}

Generally, there exists a mismatch between human and machine control, i.e. human factors result in uncertain, imprecise, and fuzzy, while machine and computer control are accurate, efficient, but unadaptable. As an artificial intelligent technique, FC is a way to make machines more intelligent enabling them to behave in a fuzzy manner like human. Proposed by Zadeh [146], FC emerged in 1965 as a tool to deal with uncertain, imprecise, or qualitative decision-making problems. As shown in Figure 12, FC utilizes linguistic information to model the qualitative aspects of human knowledge and reasoning processes without employing precise quantitative analysis. It is considered as a nonmathematical control approach for the system that cannot be well defined or modelled [147], and has been extensively used in the applications of UMS control. In general, FC has heuristics-based and model-based approaches, and the later (e.g. Takagi-Sugeno modeledbased approach) is more popular. There are plenty of research papers using model-based approaches which readers can refer to, e.g. for set-point regulation [27, 148, 149, 150, 151], for tracking control $[152,153,154,93,155]$, and for FC combining with some other control techniques $[156,157,158,159]$.

\subsection{Optimal control}

Strictly speaking, optimal control is a control problem rather than a method, but the authors would refer it to different control approaches for optimization in this paper. The objective of optimal control is to find a control law $u(t)$ that minimizes (or maximizes) the cost function

$$
J=\int f(q(t), \dot{q}(t), u(t)) \cdot d t
$$

Optimal control problems mainly can be considered into two categories: energy-optimal and time-optimal. For energy-optimal control, the issue concerns the energy consumption of the system, e.g. [160, 161, 162, 163]. For time-optimal control, most of the control 
approaches were based on differential geometric method for optimal control problems of either a class of nonholonomic rigid systems $[164,165,166,167]$ or some specific applications [168, 169], or even, many different approaches have been developed for a same problem, e.g. spacecraft maneuvers $[170,171,172]$. Similar to energy-optimal control, there has not been a general approach for time-optimal control of UMS. Therefore, a general optimal control theory for UMS like the linear optimal control theory would be appreciated.

\section{Current challenges of UMS}

Highly nonlinear characteristics and reduced dimension of the input space are intractable issues for controlling UMS. The control methods were designed either to adapt to nonlinearities or to reduce the system order to a low-dimensional model. But not all these methods are practically applicable due to system constraints (e.g. actuator power limitation). This section reviews these issues from the points of view of theoretical challenge and practical applicability.

\subsection{Theoretical challenges}

Controllability and stabilization: As a class of strongly nonlinear systems, interest in UMS always concentrates on its complex dynamics, nonholonomic constraint, and lack of feedback linearization which classical linear control theory is not applicable. Although the dynamics of UMS has been well understood, more concerns have been made on its controllability. Some early works $[173,174]$ have shown that the controllability of a system is dependent on its linearization property, i.e. a system is locally controllable if its linearization at an equilibrium is controllable. Then these studies were mainly limited to nonholonomic systems satisfying non-integrable kinematics relations $[1,175]$ with an extension to the UMSs that had non-integrable dynamics [176]. The important work done by Reyhanoglu et al. [176] has showed that strong accessibility of UMS is a necessary condition for small-time local controllability [173] at an equilibrium, but it is insufficient 
to prove that an asymptotically feedback stabilization exists. Luca et al. [12] discussed sufficient conditions of small-time local controllability for UMS which suggested to use either a discontinuous or a time-varying feedback controller for stabilization as timeinvariant continuous feedback control was not applicable for UMS, e.g. [97, 177]. This more or less explains the reason that discontinuous control methods (e.g. sliding mode control) are of great interests by researchers at present.

Recent works on the stabilization of UMS using discontinuous feedback control have been developed for some specific UMSs or a specific class of UMSs rather than the entire framework. For example, Xu and Özgüner [71] proposed a global stabilization scheme for a class of UMSs in cascaded form. Sankaranarayanan and Mahindrakar [143] developed a control approach which can globally stabilize a class of underactuated mobile systems that were not linearly controllable and violated Brockett's necessary condition [178]. Moreover, semi-global and global stabilizations were also done in [139, 98, 44, 179] for both set-point regulation and trajectory tracking control problems. A special concern is paid to the setpoint regulation of UMS as there is no breakthrough on global stabilization, and almost all the works (e.g. $[3,28,16,33])$ were done by supervisory switching from swing-up control to balancing control which was actually not a global stabilizer. Global stability results maybe only exist for a limited class of UMSs. Thus, it is recommended to focus on specific classes of UMSs or a single UMS in order to make progress, e.g. [44, 20, 87, 180].

Configuration characteristics: A notable work indebted to Olfati-Saber [10] who proposed an explicit change of coordinates that transformed several classes of UMSs into cascade nonlinear systems with structural properties. The work was carried out under the configurations of UMS with actuated/unactuated shape variables, input coupling, and non-integrable momentums which had led to a detailed classification in Table 3 . The merit of this work is that UMSs can be explicitly transformed into a strict feedback form, feedforward form, and non-triangular linear/quadratic form based on their configuration characteristics for which relevant control methods can be applied to easily. Some example applications of the work can be found in $[101,66,87,181,182]$. 
The constraint of UMS is another configuration characteristic that should draw reader's attention. As shown in Table 2, UMSs can be classified into holonomic and nonholonomic based on the integrability of their constraints. Almost all the research of UMS so far is on control of nonholonomic UMSs. However, dramatically rising interests have been paid to a class of holonomic underactuated robots $[100,35,183,184,185,37,186]$ recently due to their broad applications, and they have brought many interesting control issues. An example of this type is illustrated here to discuss some new control issues. An underactuated robot, so-called Capsubot, is shown in Fig. 13, where an inner mass $m_{2}$ interacts with a capsule main body $m_{1}$ through an internal force $u$ in the presence of dry friction with static friction coefficient $\mu$. The dynamic model of the system can be written as

$$
\left[\begin{array}{cc}
m_{1} & m_{2} \\
0 & m_{2}
\end{array}\right]\left[\begin{array}{l}
\ddot{x}_{1} \\
\ddot{x}_{2}
\end{array}\right]+\left[\begin{array}{c}
\mu\left(m_{1}+m_{2}\right) g \cdot \operatorname{sign}\left(\dot{x}_{1}\right) \\
0
\end{array}\right]=\left[\begin{array}{l}
0 \\
u
\end{array}\right]
$$

where $g$ is the acceleration due to gravity. The Capsubot is a flat UMS with 2-DOF and a single control input, and it is also holonomic as its second-order constraint is completely integrable when $\dot{x}_{1} \neq 0$. The control task of the system is to drive the entire system to a desired equilibrium $\left(x_{e}^{1}, x_{e}^{2}\right)$ which can be considered as a control problem of set-point regulation. Then the issue emerges due to its non-smooth friction term which raises the difficulty level of control. On the other hand, this term is vital, because the system will not progress without it. Up to now, only open-loop control [183] and semi-closed-loop control [100] (i.e. closed-loop control of subsystem) are available, and the closed-loop control of UMS with non-smooth dynamics is still an open issue. Some recent works can be referred to $[98,187,36,37]$.

\subsection{Practical challenges}

Industrial needs: Nowadays, UMSs are widely used in aerospace and industry for exploration and manufacture. Examples of these systems include ship, underwater vehicle, helicopter, aircraft, satellite, mobile robot, space platform, and flexible joint robot, etc. Although these examples have received many well-established successes in well-structured 
environment, their control techniques for complex uncertain environment are still immature $[7,8]$. However, there is always a desiderative expectation of new applications of UMSs that requires autonomous operation in an unstructured and possibly dynamically changing environment [188]. Thus, control techniques that satisfy these needs become crucial.

High-DOF complex UMS: The benefits brought by UMS can be reduced weight, cost, and energy consumption, as well as maintaining adequate degrees of dexterity without losing reachable configuration space. They also have no or less damage when hitting an object, and more tolerance for actuator failure. As the DOF of UMS increases, the reliability of the system declines which results in practical problem of control design. Examples of such high-DOF complex systems include the systems involving an extremely large number of control loops, the coordination of a large number of autonomous agents to control a nonlinear hybrid stochastic system, or very large model uncertainties [7]. These systems cannot work under hazardous or unsafe environment without any considerable reliability, since the costs of damage and loss are extremely high. Robustness to noise, disturbance, and uncertainty secures the reliability of UMS, and a need of new tools for addressing robustness issue is highly desirable.

Fault tolerant detection and control: The failures of actuator and sensor may cause a practical problem, as the failure of actuators causes the reduction of the number of control inputs which converts a fully-actuated system to a UMS [189], or converts a UMS with more control inputs to a UMS with less control inputs. The failure of sensors results in delayed or missing the feedback signal which affects the overall tracking performance of the system. Therefore, practical failures of actuators and sensors are to be considered for scientists and engineers in the design of UMS, and a fault detection mechanism is highly recommended, e.g. [190, 191, 192, 193].

Networked UMS: Another practical concern is networked control of UMS. Once network is introduced, communication package delay or loss may lead to poor control performance, since the communication between actuator and sensor can be interrupted. Control 
of UMS should be robust to network delay, and a network control technique (e.g. predictive control) is required.

\section{Concluding Remarks and Future Research}

UMS is such an important class of mechanical systems that have been applied in diverse engineering applications. Control of UMS is one of the most active fields of research in control community. For such a wide research area, it is difficult to include all the works of UMS in a review paper. However, we believe that this survey could provide the reader with the most up-to-date knowledge in the field of UMS. Finally, several important issues and possible future research directions are drawn:

- Set-point regulation: As a mature and well-defined control issue, set-point regulation for a class of UMSs, such as the Acrobot, the Pendubot, and the cart-pole system, has been extensively studied. However, there are still potential contributions for this matter, e.g. recent works $[21,168,194,22,195]$, which proposed new set-point regulation methods;

- Tracking control: Compared to fruitful achievements for fully-actuated systems, tracking control of UMS is still a challenge topic. Some recent works $[143,71,179]$ can be referred;

- Modelling with friction: Though friction plays a significant role in some precise applications $[11,100]$, it is always ignored $[10,4]$, or simplified $[184,93]$ in modelling of UMS. Some references [98, 196, 197, 198] can give readers an open mind along this direction;

- Novel UMS: Some UMS examples, such as biped robot [199, 200], robotic hand [201], pendulum-driven cart [36, 37], Capsubot [100, 100], and AcroBOX [202], should bring reader's attention as design of new UMS is one of the trends in this field. We believe it is a promising research direction. 


\section{Acknowledgement}

The authors would like to thank anonymous reviewers for their valuable comments on this work.

\section{References}

[1] A. M. Bloch, M. Reyhanoglu, N. H. McClamroch, Control and stabilization of nonholonomic dynamic systems, IEEE Transaction on Automatic Control 37 (1992) $1746-1757$.

[2] A. Astolfi, Discontinuous control of nonholonomic system, Systems and Control Letters 27 (1996) 37-45.

[3] M. W. Spong, Control Problems in Robotics and Automation, London: SpringerVerlag, 1998, Ch. Underactuated Mechanical Systems.

[4] I. Fantoni, R. Lozano, Nonlinear control for underactuated mechanical systems, London: Springer-Verlag, 2001.

[5] H. Arai, S. Tachi, Position control of a manipulator with passive joints using dynamic coupling, IEEE Transactions on Robotics and Automation 7 (1991) 528-534.

[6] P. Baglioni, R. Fisackerly, B. Gardini, G. Gianfiglio, A. L. Pradier, A. Santovincenzo, J. L. Vago, M. van Winnendael, The Mars exploration plans of ESA, IEEE Robotics and Automation Magazine 13 (2006) 83-89.

[7] P. Bars, P. Colaneri, C. Souza, L. Dugard, F. Allgower, A. Kleimenov, C. Scherer, Theory, algorithms and technology in the design of control systems, Annual Reviews in Control 30 (2006) 19-30.

[8] A. Ollero, S. Boverie, D. Cho, H. Hashimoto, M. Tomizuka, W. Wang, D. Zuhlke, Mechatronics, robotics and componets for automation and control: IFAC milestone report, Annual Reviews in Control 30 (2006) 41-54. 
[9] K. Dutton, S. Thompson, B. Barraclough, The art of control engineering, Addison Wesley, 1997.

[10] R. Olfati-Saber, Nonlinear control of underactuated mechanical systems with application to robotics and aerospace vehicles, Ph.D. thesis, Department of Electrical Engineering and Computer, Massachusetts Institute of Technology (2001).

[11] N. P. I. Aneke, Control of underactuated mechanical systems, Ph.D. thesis, Mechanical Engineering, Eindhoven University of Technology (2003).

[12] A. de Luca, R. Mattone, G. Oriolo, Control of underactuated mechanical systems: Application to the plannar 2R robot, in: Proceedings of the Conference on Decision and Control, Kobe, Japan, 1996, pp. 1455-1460.

[13] M. Nikkhah, H. Ashrafiuon, K. R. Muske, Optimal sliding mode control for underactuated systems, in: Proceedings of the American Control Conference, Mineapolis, Minnesota, USA, 2006, pp. 4688-4693.

[14] H. Yu, S. Lloyd, Variable struture adaptive control of robot manipulators, IEE Control Theory and Applications 144 (1997) 167-176.

[15] M. W. Spong, The swing-up control problem for the Acrobot, IEEE Control System Magazine 47 (1995) 49-55.

[16] I. Fantoni, R. Lozano, M. W. Spong, Energy based control of the Pendubot, IEEE Transaction on Automatic Control 45 (2000) 725-729.

[17] M. W. Spong, Energy based control of a class of underactuated mechanical systems, in: Proceedings of the IFAC World Congress, San Francisco, CA, USA, 1996, pp. $431-435$.

[18] K. J. Åström, K. Furuta, Swinging up a pendulum by energy control, Automatica 36 (2000) 287-295. 
[19] M. Zhang, T. Tarn, A hybrid switching control strategy for nonlinear and underactuated mechanical systems, IEEE Transaction on Automatic Control 48 (2003) $1777-1782$.

[20] X. Z. Lai, J. H. She, S. X. Yang, M. Wu, Comprehensive unified control strategy for underactuated two-link manipulators, IEEE Transactions on Systems, Man, and Cybernetics - Part B: Cybernetics 39 (2009) 389-398.

[21] T. Albahkali, R. Mukherjee, T. Das, Swing-up control of the Pendubot: an impulsemomentum approach, IEEE Transactions on Robotics 25 (2009) 975-982.

[22] R. Jafari, F. B. Mathis, R. Mukherjee, Swing-up control of the Acrobot: An impulsemomentum approach, in: Proceedings of the American Control Conference, San Francisco, CA, USA, 2011, pp. 262-267.

[23] H. Yabuno, T. Matsuda, N. Aoshima, Reachable and stabilizable area of an underactuated manipulator without state feedback control, IEEE/ASME Trans Mechatronics 10 (2005) 397-403.

[24] M. W. Spong, Partial feedback linearization of underactuated mechanical systems, in: Proceedings of the IEEE/RSJ/GI International Conference on Intelligent Robots and Systems, Munich, Germany, 1994, pp. 314-321.

[25] J. Zhao, M. W. Spong, Hybrid control for global stabilization of the cart-pendulum system, Automatica 37 (2001) 1941-1951.

[26] D. Chatterjee, A. Patra, H. K. Joglekar, Swing-up and stabilization of a cartpendulum system under restricted cart track length, System Control Letter 47 (2002) 355-364.

[27] N. Muškinja, B. Tovornik, Swinging up and stabilization of a real inverted pendulum, IEEE Transaction on Industrial Electronics 53 (2006) 631-639. 
[28] R. Fierro, F. L. Lewis, A. Lowe, Hybrid control for a class of underactuated mechanical systems, IEEE Transaction on Systems, Man, and Cybernetics - Part A: Systems and Humans 29 (1999) 649-654.

[29] K. Pathak, J. Franch, S. K. Agrawal, Velocity and position control of a wheeled inverted pendulum by partial feedback linearization, IEEE Trans Robotics 21 (2005) $505-513$.

[30] Y. Kim, S. H. Kim, Y. K. Kwak, Dynamic analysis of a nonholonomic two-wheeled inverted pendulum robot, J Intelligent and Robotic Systems 44 (2005) 25-46.

[31] J. Huang, Z. H. Guan, T. Matsuno, T. Fukuda, K. Sekiyama, Sliding-mode velocity control of mobile-wheeled inverted-pendulum systems, IEEE Transactions on Robotics 26 (2010) 750-758.

[32] X. Xin, M. Kaneda, Analysis of the energy-based control for swinging up two pendulums, IEEE Transactions on Automatic Control 50 (2005) 679-684.

[33] W. Zhong, H. Rock, Energy and passivity based control of the double inverted pendulum on a cart, in: Proceedings of the IEEE International Conference on Control Applications, Mexico City, Mexico, 2001, pp. 896-901.

[34] N. A. Chaturvedi, N. H. McClamroch, D. S. Bernstein, Asymptotic smooth stabilization of the inverted 3-D pendulum, IEEE Trans Automatic Control 54 (2009) $1204-1215$.

[35] H. Li, K. Furuta, F. L. Chernousko, A pendulum-driven cart via internal force and static friction, in: Proceedings of IEEE International Conference on Physics and Control, St. Petersburgh, Russia, 2005, pp. 15-17.

[36] H. Yu, Y. Liu, T. C. Yang, Closed-loop tracking control of a pendulum-driven cartpole underactuated system, Proceedings of the Institution of Mechanical Engineers, Part I: Journal of Systems and Control Engineering 222 (2008) 109-125. 
[37] Y. Liu, H. Yu, S. Cang, Modelling and motion control of a double-pendulum driven cart, Proceedings of the Institution of Mechanical Engineers, Part I: Journal of Systems and Control Engineering 226 (2012) 175-187.

[38] Y. Fang, W. Dixon, D. Dawson, E. Zergeroglu, Nonlinear coupling control laws for an underactuated overhead crane system, IEEE/ASME Transactions on Mechatronics 8 (2003) 418-423.

[39] B. Ma, Y. Fang, Y. Zhang, Switching-based emergency braking control for an overhead crane system, IET Control Theory and Applications 4 (2010) 1739-1747.

[40] Y. Fang, B. Ma, P. Wang, X. Zhang, A motion planning-based adaptive control method for an underactuated crane system, IEEE Transactions on Control Systems Technology 20 (2012) 241-248.

[41] N. Sun, , Y. Fang, Y. Zhang, B. Ma, A novel kinematic coupling-based trajectory planning method for overhead cranes, IEEE/ASME Trans Mechatronics 17 (2012) $166-173$.

[42] I. Fantoni, R. Lozano, Stabilization of the Furuta pendulum around its homoclinic orbit, Int J Control 75 (2002) 390-398.

[43] G. Viola, R. Ortega, R. Banavar, J. A. Acosta, A. Astolfi, Total energy shaping control of mechanical systems: Simplifying the matching equations via coordinate changes, IEEE Trans Automatic Control 52 (2007) 1093-1099.

[44] M. S. Park, D. K. Chwa, Swing-up and stabilization control of inverted-pendulum systems via coupled sliding-mode control method, IEEE Transaction on Industrial Electronics 56 (2009) 3541-3555.

[45] D. E. Chang, Stabilizability of controlled Lagrangian systems of two degrees of freedom and one degree of under-actuation by the energy-shaping method, IEEE Trans Automatic Control 55 (2010) 1888-1893. 
[46] A. S. Shiriaev, L. B. Freidovich, A. Robertsson, R. Johansson, A. Sandberg, Virtualholonomic-constraints-based design of stable oscillations of Furuta pendulum: Theory and experiments, IEEE Trans Robotics 23 (2007) 827-832.

[47] L. Freidovich, A. Shiriaev, F. Gordillo, F. Gómez-Estern, J. Aracil, Partial-energyshaping control for orbital stabilization of high-frequency oscillations of the Furuta pendulum, IEEE Trans Control Systems Technology 17 (2009) 853-858.

[48] R. Ortega, M. W. Spong, F. Gómez-Estern, G. Blankenstein, Stabilization of a class of underactuated mechanical systems via interconnection and damping assignment, IEEE Transactions on Automatic Control 47 (2002) 1218-1232.

[49] H. Ye, H. Wang, H. Wang, Stabilization of a PVTOL aircraft and an inertia wheel pendulum using saturation technique, IEEE Trans Control Systems Technology 15 (2007) 1143-1150.

[50] H. Ye, W. Gui, Z. P. Jiang, Backstepping design for cascade systems with relaxed assumption on Lyapunov functions, IET Control Theory and Applications 5 (2011) $700-712$.

[51] M. Lopez-Martinez, J. A. Acosta, J. M. Cano, Non-linear sliding mode surfaces for a class of underactuated mechanical systems, IET Control Theory and Applications 4 (2010) 2195-2204.

[52] T. C. Yang, A case study of magnetic suspension control, in: Proceedings of the International Conference on Control, Exeter, UK, 1996, pp. 603-607.

[53] B. V. Jayawant, T. C. Yang, S. Tollner, Control of magnetic suspension systems by maintaining continuous equilibrium points, in: Proceedings of the 14th World Congress of IFAC, Beijing, China, 1999, pp. 471-478.

[54] H. Yu, T. C. Yang, D. Rigas, B. V. Jayawant, Modelling and control of magnetic sus- 
pension systems, in: Proceedings of the IEEE International Conference on Control Applications, Glasgow, Scotland, UK, 2002, pp. 944-949.

[55] J. Hauser, S. Sastry, P. Kokotovico, Nonlinear control via approximate input-output linearization: the beam and ball example, IEEE Transaction on Automatic Control 37 (1992) 392-398.

[56] F. Andreeva, D. Aucklyb, S. Gosavic, L. Kapitanskib, A. Kelkard, W. Whitec, Matching, linear systems, and the ball and beam, Automatica 38 (2002) 2147-2152.

[57] L. Marton, A. S. Hodel, B. Lantos, J. Y. Hung, Underactuated robot control: comparing LQR subspace stabilization, and combined error metric approaches, IEEE Transactions on Industrial Electronics 50 (2008) 3724-3730.

[58] M. T. Ravichandran, A. Mahindrakar, Robust stabilization of a class of underactuated mechanical systems using time scaling and Lyapunov redesign, IEEE Trans Industrial Electronics 58 (2011) 4299-4313.

[59] C. J. Wan, D. S. Bernstein, V. T. Coppola, Global stabilization of the oscillating eccentric rotor, in: Proceedings of the IEEE International Conference on Decision and Control, Orlando, USA, 1994, pp. 4024-4029.

[60] M. Jankovic, D. Fontaine, P. V. Kokotovic, TORA example: cascade and passivitybased control designs, IEEE Transaction on Control Systems Technology 4 (1996) 292-297.

[61] Z. P. Jiang, D. J. Hill, Y. Guo, Stabilization and tracking via output feedback for the nonlinear benchmark system, Automatica 34 (1998) 907-915.

[62] Z. P. Jiang, I. Kanellakopoulos, Global output-feedback tracking for a benchmark nonlinear system, IEEE Transaction on Automatic Control 45 (2000) 1023-1027. 
[63] A. Pavlov, B. Janssen, N. van de Wouw, H. Nijmeijer, Experimental output regulation for a nonlinear benchmark system, IEEE Trans Control Systems Technology 15 (2007) 786-793.

[64] Y. F. Chen, A. C. Huang, Controller design for a class of underactuated mechanical systems, IET Control Theory and Applications 6 (2012) 103-110.

[65] J. Hauser, S. Sastry, G. Meyer, Nonlinear control design for slightly non-minimum phase systems, Automatica 28 (1992) 665-679.

[66] R. Olfati-Saber, Global configuration stabilization for the VTOL aircraft with strong input coupling, IEEE Trans Automatic Control 47 (2002) 1949-1952.

[67] P. Setlur, D. Dawson, Y. Fang, B. Costic, Nonlinear tracking control of the VTOL aircraft, in: Proceedings of the 40th IEEE Conference on Decision and Control, Orlando, USA, 2001, pp. 4592-4597.

[68] A. Zavala-Rio, I. Fantoni, R. Lozano, Global stabilization of a PVTOL aircraft model with bounded inputs, Int J Control 76 (2003) 1833-1844.

[69] R. Lozano, P. Castillo, A. Dzul, Global stabilization of the PVTOL: real-time application to a mini-aircraft, Int J Control 77 (2004) 735-740.

[70] R. Wood, B. Cazzolato, An alternative nonlinear control law for the global stabilization of the PVTOL vehicle, IEEE Trans Automatic Control 52 (2007) 1282-1287.

[71] R. Xu, U. Özgüner, Sliding mode control of a class of underactuated systems, Automatica 44 (2008) 233-241.

[72] P. Martin, S. Devasia, B. Paden, A different look at output tracking: control of a VTOL aircraft, Automatica 32 (1996) 101-107.

[73] F. Lin, W. Zhang, R. D. Brandt, Robust hovering control of a PVTOL aircraft, IEEE Transactions on Control Systems Technology 7 (1999) 343-351. 
[74] S. A. Al-Hiddabi, N. H. McClamroch, Tracking and maneuver regulation control for nonlinear non-minimum phase systems: application to flight control, IEEE Transactions on Control Systems Technology 10 (2002) 780-792.

[75] L. Marconi, A. Isidori, A. Serrani, Autonomous vertical landing on an oscillating platform: an internal-model based approach, Automatica 38 (2002) 21-32.

[76] K. D. Do, Z. P. Jiang, J. Pan, On global tracking control of a VTOL aircraft without velocity measurements, IEEE Trans Automatic Control 48 (2003) 2212-2217.

[77] G. Notarstefano, J. Hauser, R. Frezza, Trajectory manifold exploration for the PVTOL aircraft, in: Proceedings of the IEEE conference on decision and control and european control conference, Seville, Spain, 2005, pp. 5848-5853.

[78] L. Consolini, M. Tosques, On the VTOL exact tracking with bounded internal dynamics via a Poincaré map approach, IEEE Trans Automatic Control 52 (2007) $1757-1762$.

[79] L. Consolini, M. Maggiore, C. Nielsen, M. Tosques, Path following for the PVTOL aircraft, Automatica 46 (2010) 1284-1296.

[80] A. Behal, D. M. Dawson, W. E. Dixon, Y. Fang, Tracking and regulation control of an underactuated surface vessel with nonintegrable dynamics, IEEE Trans Automatic Control 47 (2002) 495-500.

[81] K. Y. Pettersen, F. Mazenc, H. Nijmeijer, Global uniform asymptotic stabilization of an underactuated surface vessel: Experimental results, IEEE Trans Control Systems Technology 12 (2004) 891-903.

[82] W. Dong, Y. Guo, Global time-varying stabilization of underactuated surface vessel, IEEE Trans Automatic Control 50 (2005) 859-864. 
[83] M. Reyhanoglu, A. Bommer, Tracking control of an underactuated autonomous surface vessel using switched feedback, in: Proceedings of the 32nd Annual Conference on Industrial Electronics, Daytona Beach, USA, 2006, pp. 3833-3838.

[84] J. Ghommam, F. Mnif, A. Benali, N. Derbel, Asymptotic backstepping stabilization of an underactuated surface vessel, IEEE Trans Control Systems Technology 14 (2006) 1150-1157.

[85] H. Ashrafiuon, K. R. Muske, L. C. McNinch, R. A. Soltan, Sliding-mode tracking control of suerface vessels, IEEE Transactions on Industrial Eletronics 55 (2008) 4004-4012.

[86] W. Dong, J. A. Farrell, Formation control of multiple underactuated surface vessels, IET Control Theory and Applications 2 (2008) 1077-1085.

[87] J. Ghommam, F. Mnif, N. Derbel, Global stabilisation and tracking control of underactuated surface vessels, IET Control Theory Appl 4 (2010) 71-88.

[88] A. P. Aguiar, J. P. Hespanha, Trajectory-tracking and path-following of underactuated autonomous vehicles with parametric modeling uncertainty, IEEE Trans Automatic Control 52 (2007) 1362-1379.

[89] F. Fahimi, Sliding-mode formation control for underactuated surface vessels, IEEE Trans Robotics 23 (2007) 617-622.

[90] R. Yu, Q. Zhu, G. Xia, Z. Liu, Sliding mode tracking control of an underactuated surface vessel, IET Control Theory and Applications 6 (2012) 461-466.

[91] O. Egeland, M. Dalsmo, O. J. Sordalen, Feedback control of an nonholonomic underwater vehicle with constant desired configuration, International Journal of Robotics Research 15 (1996) 24-35. 
[92] M. C. Deng, A. Inoue, Y. Shibata, K. Sekiguchi, N. Ueki, An obstacle avoidance method for two wheeled mobile robot, in: Proceedings of the IEEE International Conference on Networking, Sensing and Control, London, UK, 2007, pp. 689-692.

[93] J. Velagic, B. Lacevic, N. Osmic, Motion Planning, InTech Education and Publishing, 2008, Ch. Nonlinear motion control of mobile robot dynamic model.

[94] G. C. Walsh, R. Montgomery, S. Sastry, Orientation control of the dynamic satellite, in: Proceedings of the American Control Conference, Berkeley, CA, USA, 1994, pp. $138-142$.

[95] F. Bullo, Nonlinear control of mechanical systems: A Riemannian geometry approach, Ph.D. thesis, Department of Control and Dynamical Systems, California Institute of Technology (1998).

[96] A. de Luca, B. Siciliano, Trajectory control of a non-linear one-link fexible arm, International Journal of Control 50 (1989) 1699-1715.

[97] G. Oriolo, T. Nakamura, Control of mechanical systems with second-order nonholonomic contstraints: Underactuated manipulators, in: Proceedings of the IEEE International Conference on Decision and Control, Brighton, UK, 1991, pp. 23982403.

[98] R. Martinez, J. Alvarez, Y. Orlov, Hybrid sliding-mode-based control of underactuated systems with dry frictions, IEEE Transaction on Industrial Electronics 55 (2008) 3998-4003.

[99] R. M. A. de Luca, S. Iannitti, G. Oriolo, Control problems in underactuated manipulators, in: Proceedings of the IEEE/ASME International Conference on Advanced Intelligent Mechatronics, Como, Italy, 2001, pp. 8-12.

[100] Y. Liu, H. Yu, T. C. Yang, Analysis and control of a Capsubot, in: Proceedings of IFAC World Congress, Seoul, Korea, 2008, pp. 756-761. 
[101] R. Olfati-Saber, Normal forms for underactuated mechanical systems with symmetry, IEEE Transactions on Automatic Control 47 (2002) 305-308.

[102] M. Zhang, T. Tarn, Hybrid control of the Pendubot, IEEE/ASME Transactions on Mechatronics 7 (2002) 79-86.

[103] R. Olfati-Saber, Trajectory tracking for a flexible one-link robot using a nonlinear noncollocated output, in: Proceedings of the IEEE Conference on Decision and Control, Sydney, Australia, 2000, pp. 4024-4029.

[104] K. Y. Pettersen, H. Nijmeijer, Tracking control of an underactuated surface vessel, in: Proceedings of the IEEE Conference on Decision and Control, Tampa, Florida, USA, 1998, pp. 4561-4566.

[105] L. C. McNich, H. Ashrafiuon, K. R. Muske, Sliding mode setpoint control of an underactuated surface vessel: simulation and experiment, in: Proceedings of the American Control Conference, Baltimore, MD, USA, 2010, pp. 5212-5217.

[106] A. Shiriaev, H. Ludvigsen, O. Egeland, A. Pogromsky, On global properties of passivity based control of the inverted pendulum, in: Proceedings of the 38th Conference on Decision and Control, Phoenix, Arizona, USA, 1999, pp. 2513-2518.

[107] B. Gao, X. Zhang, H. Chen, J. Zhao, Energy-based control design of an underactuated 2-dimensional TORA system, in: Proceedings of the IEEE/RSJ International Conference on Intelligent Robots and Systems, St. Louis, USA, 2009, pp. 1296-1301.

[108] M. W. Spong, J. K. Holm, D. Lee, Passivity-based control of bipedal locomotion, IEEE Robotics and Automation Magazine 14 (2007) 30-40.

[109] A. van der Schaft, $L_{2}$-gain analysis of nonlinear systems and nonlinear state feedback $H_{\infty}$ control, IEEE Trans Automatic Control 37 (1992) 770-784.

[110] A. van der Schaft, $L_{2}$-Gain and Passivity in Nonlinear Control, New York: SpringerVerlag, 1999. 
[111] Z. P. Jiang, Global tracking control of underactuated ships by Lyapunovs direct method, Automatica 38 (2002) 301-309.

[112] W. N. White, M. Foss, X. Guo, A direct Lyapunov approach for a class of underactuated mechanical systems, in: Proceedings of the American Control Conference, Minneapolis, Minnesota, USA, 2006, pp. 103-110.

[113] P. V. Kokotovic, M. Krstic, I. Kmellakopoulos, Backstepping to passivity: Recursive design of adaptive systems, in: Proceedings of the 31st Conference on Decision and Control, Tucson, Arizona, USA, 1992, pp. 3276-3280.

[114] K. D. Do, Z. P. Jiang, J. Pan, Underactuated ship global tracking under relaxed conditions, IEEE Trans Automatic Control 47 (2002) 1529-1536.

[115] P. Batista, C. Silvestre, P. Oliveira, A sensor-based controller for homing of underactuated AUVs, IEEE Trans Robotics 25 (2009) 701-716.

[116] F. Y. Bi, Y. J. Wei, J. Z. Zhang, W. Cao, Position-tracking control of underactuated autonomous underwater vehicles in the presence of unknown ocean currents, IET Control Theory Appl 4 (2010) 2369-2380.

[117] D. Chwa, Global tracking control of underactuated ships with input and velocity constraints using dynamic surface control method, IEEE Trans Control Systems Technology 19 (2011) 1357-1370.

[118] A. Abdessameuda, A. Tayebi, Global trajectory tracking control of VTOL-UAVs without linear velocity measurements, Automatica 46 (2010) 1053-1059.

[119] A. Gruszka, M. Malisoff, F. Mazenc, On tracking for the PVTOL model with bounded feedbacks, in: Proceedings of the American Control Conference, San Francisco, CA, USA, 2011, pp. 1428-1433. 
[120] R. Ortega, A. van der Schaftb, B. Maschkec, G. Escobar, Interconnection and damping assignment passivity-based control of port-controlled Hamiltonian systems, Automatica 38 (2002) 585-596.

[121] R. Ortega, E. Canseco, Interconnection and damping assignment passivity-based control: A survey, European J Control 10 (2004) 432-450.

[122] A. M. Bloch, N. E. Leonard, J. E. Marsden, Controlled Lagrangians and the stabilization of mechanical systems I: The first matching theorem, IEEE Trans Automatic Control 45 (2000) 2253-2270.

[123] A. M. Bloch, D. E. Chang, N. E. Leonard, J. E. Marsden, Controlled Lagrangians and the stabilization of mechanical systems II: Potential shaping, IEEE Trans Automatic Control 46 (2001) 1556-1571.

[124] M. W. Spong, On the robust control of robot manipulators, IEEE Transactions on Automatic Control 27 (1992) 1782-1786.

[125] K. J. Åström, B. Wittenmark, Adaptive Control, Addison-Wesley, 1995.

[126] Y. L. Gu, A direct adaptive control scheme for underactuated dynamic systems, in: Proceedings of the Conference on Decision and Control, San Antonio, Texas, USA, 1993, pp. 1625-1627.

[127] J. H. Shin, Trajectory planning and robust adaptive control for underactuated manipulators, Eletronics Letters 34 (1998) 1705-1706.

[128] C. Y. Su, Y. Stepanenko, Adaptive variable structure set-point control of underactuated robots, IEEE Transaction on Automatic Control 44 (1999) 2090-2093.

[129] K. D. Do, Z. P. Jiang, J. Pan, Robust adaptive path following of underactuated ships, Automatica 40 (2004) 929-944. 
[130] J. E. Slotine, Sliding controller design for nonlinear systems, International Journal of Control 40 (1984) 421-434.

[131] H. Ashrafiuon, R. S. Erwin, Shape change maneuvers for attitude control of underactuated satellites, in: Proceedings of the American Control Conference, Portland, OR, USA, 2005, pp. 895-900.

[132] L. C. McNinch, H. Ashrafiuon, Predictive and sliding mode cascade control for unmanned surface vessels, in: Proceedings of the American Control Conference, San Francisco, CA, USA, 2011, pp. 184-189.

[133] H. Ríos, A. Rosales, A. Ferreira, A. Dávila, Robust regulation for a 3-DOF helicopter via sliding-modes control and observation techniques, in: Proceedings of the American Control Conference, Baltimore, MD, USA, 2010, pp. 4427-4432.

[134] N. B. Almutairi, M. Zribi, On the sliding mode control of a ball on a beam system, Nonlinear Dynamics 59 (2010) 221-238.

[135] D. C. DiFiore, Sliding mode control applied to an underactuated fuel cell system (2009).

[136] J. M. Avis, S. G. Nersesov, R. Nathan, H. Ashrafiuon, K. R. Muske, A comparison study of nonlinear control techniques for the RTAC system, Nonlinear Analysis: Real World Applications 11 (2010) 2647-2658.

[137] M. Nikkhah, H. Ashrafiuon, F. Fahimi, Robust control of underactuated bipeds using sliding modes, Robotica 25 (2007) 367-374.

[138] N. B. Almutairi, M. Zribi, Sliding mode control of a three-dimensional overhead crane, Journal of Vibration and Control 15 (2009) 1679-1730.

[139] W. Wang, J. Yi, D. Zhao, D. Liu, Design of a stable sliding-mode controller for a class of second-order underactuated systems, IEE Procceding on Control Theory Applications 151 (2004) 683-690. 
[140] H. Ashrafiuon, R. S. Erwin, Sliding mode control of underactuated multibody systems and its application to shape change control, International Journal of Control $81(2008)$ 1849-1858.

[141] D. W. Qian, J. Q. Yi, D. B. Zhao, Robust control using sliding mode for a class of underactuated systems with mismatched uncertainties, in: Proceedings of the IEEE International Conference on Robotics and Automation, Rome, Italy, 2007, pp. 1449-1454.

[142] M. Yue, P. Hu, W. Sun, Path following of a class of non-holonomic mobile robot with underactuated vehicle body, IET Control Theory and Applications 4 (2010) $1898-1904$.

[143] V. Sankaranarayanan, A. D. Mahindrakar, Control of a class of underactuated mechanical systems using sliding modes, IEEE Transaction on Robotics 25 (2009) 459-467.

[144] S. G. Nersesov, H. Ashrafiuon, P. Ghorbanian, On the stability of sliding mode control for a class of underactuated nonlinear systems, in: Proceedings of American Control Conference, Baltimore, MD, USA, 2010, pp. 3446-3451.

[145] K. D. Young, V. I. Utkin, Ü. Özgüner, A control engineer's guide to sliding mode control, IEEE Transactions on Control Systems Technology 7 (1999) 328-342.

[146] L. A. Zadeh, Fuzzy sets, Information and Control 8 (1965) 338-353.

[147] K. Michels, F. Klawonn, R. Kruse, A. Numberger, Fuzzy Control: Fundamentals, Stability and Design of Fuzzy Controllers, New York: Springer-Verlag, 2006.

[148] M. I. EI-Hawwary, A. L. Elshafei, H. M. Emara, H. A. Fattah, Adaptive fuzzy control of the inverted pendulum problem, IEEE Transactions on Control System Technology 14 (2006) 1135-1144. 
[149] K. Ichida, K. Watanabe, K. Izumi, N. Uchida, Fuzzy switching control of underactuated manipulators with approximated switching regions, in: Proceedings of the IEEE/RSJ International Conference on Intelligent Robots and Systems, Beijing, China, 2006, pp. 586-591.

[150] C. W. Tao, J. S. Taur, T. W. Hsieh, C. L. Tsa, Design of a fuzzy controller with fuzzy swing-up and parallel distributed pole assignment schemes for an inverted pendulum and cart system, IEEE Transactions on Control System Technology 16 (2008) $1277-1288$.

[151] R. J. Wai, M. A. Kuo, J. D. Lee, Cascade direct adaptive fuzzy control design for a nonlinear two-axis inverted-pendulum servomechanism, IEEE Transactions on Systems, Man, and Cybernetics - Part B: Cybernetics 38 (2008) 439-454.

[152] O. Begovich, E. N. Sanchez, M. Maldonado, Takagi-Sugeno fuzzy scheme for realtime trajectory tracking of an underactuated robot, IEEE Transaction on Control System Technology 10 (2002) 14-20.

[153] Z. Cai, C. Y. Su, Real-time tracking control of underactuated pendubot using Takagi-Sugeno fuzzy systems, in: Proceedings of the IEEE International Symposium on Computational Intelligence in Robotics and Automation, Kobe, Japan, 2003, pp. $73-78$.

[154] W. Li, K. Tanaka, H. O. Wang, Acrobatic control of a Pendubot, IEEE Transaction on Fuzzy Systems 12 (2004) 549-552.

[155] S. M. Raguraman, D. Tamilselvi, N. Shivakumar, Mobile robot navigation using fuzzy logic controller, in: Proceedings of the International Conference on Control, Automation, Communication and Energy Conservation, Perundurai, Tamilnadu, India, 2009, pp. 1-5.

[156] C. L. Hwang, H. M. Wu, C. L. Shih, Fuzzy sliding-mode underactuated control for 
autonomous dynamic balance of an electrical bicycle, IEEE Transaction on Control System Technology 17 (2009) 658-670.

[157] T. Q. Vinh, N. H. Giap, T. W. Kim, M. G. Jeong, J. H. Shin, W. H. Kim, Adaptive robust fuzzy control and implementation for path tracking of a mobile robot, in: Proceedings of the IEEE International Symposium on Industrial Electronics, Seoul, Korea, 2009, pp. 1943-1949.

[158] C. C. Kung, T. H. Chen, L. C. Huang, Adaptive fuzzy sliding mode control for a class of underactuated systems, in: Proceedings of the IEEE International Conference on Fuzzy Systems, Taipei, Taiwan, 2009, pp. 1791-1796.

[159] S. Aloui, O. Pagès, A. Hajjaji, A. Chaari, Y. Koubaa, Robust adaptive fuzzy sliding mode control design for a class of MIMO underactuated systems, in: Proceedings of the IFAC World Congress, Milano, Italy, 2011, pp. 11127-11132.

[160] I. Duleba, J. Z. Sasiadek, Nonholonomic motion planning based on Newton algorithm with energy optimization, IEEE Trans Control Systems Technology 11 (2003) $355-363$.

[161] F. Plestan, J. W. Grizzle, E. R. Westervelt, G. Abba, Stable walking of a 7-DOF biped robot, IEEE Trans Robotics and Automation 19 (2003) 653-668.

[162] E. C. Yang, P. C. Chao, C. K. Sung, Optimal control of an under-actuated system for landing with desired postures, IEEE Trans Control Systems Technology 19 (2011) $248-255$.

[163] D. Tlalolini, C. Chevallereau, Y. Aoustin, Human-like walking: Optimal motion of a bipedal robot with toe-rotation motion, IEEE Trans Robotics 16 (2011) 310-320.

[164] F. Bullo, K. M. Lynch, Kinematic controllability for decoupled trajectory planning in underactuated mechanical systems, IEEE Trans Robotics and Automation 17 (2001) 402-412. 
[165] C. Park, D. J. Scheeres, V. Guibout, A. Bloch, Global solution for the optimal feedback control of the underactuated Heisenberg system, IEEE Trans Automatic Control 53 (2008) 2638-2642.

[166] M. B. Kobilarov, J. E. Marsden, Discrete geometric optimal control on Lie groups, IEEE Trans Robotics 27 (2011) 641-655.

[167] I. Hussein, A. M. Bloch, Optimal control of underactuated nonholonomic mechanical systems, IEEE Transaction on Automatic Control 53 (2008) 668-682.

[168] M. B. P. Mason, B. Piccoli, Time optimal swing-up of the planar pendulum, IEEE Transaction on Automatic Control 53 (2008) 1876-1886.

[169] P. Paoletti, R. Genesio, Rate limited time optimal control of a planar pendulum, Systems and Control Letters 60 (2011) 264-270.

[170] M. Krstic, P. Tsiotras, Inverse optimal stabilization of a rigid spacecraft, IEEE Trans Automatic Control 44 (1999) 1042-1049.

[171] P. Tsiotras, J. Luo, Control of underactuated spacecraft with bounded inputs, Automatica 36 (2000) 1153-1169.

[172] R. Sharma, A. Tewari, Optimal nonlinear tracking of spacecraft attitude maneuvers, IEEE Trans Control Systems Technology 12 (2004) 677-682.

[173] H. J. Sussmann, A general theorem on local controllability, SIAM J Control Optim 25 (1987) 158-194.

[174] R. M. Bianchini, G. Stefani, Controllability along a trajectory: a variational approach, SIAM Journal of Control and Optimization 31 (1993) 900-927.

[175] I. Kolmanovsky, N. H. McClamroch, Developments in nonholonomic control problems, IEEE Contr Syst Mag 15 (1995) 20-36. 
[176] M. Reyhanoglu, A. Schaft, N. H. McClamroch, I. Kolmanovsky, Dynamics and control of a class of underactuated mechanical systems, IEEE Transaction on Automatic Control 44 (1999) 1663-1671.

[177] K. Y. Wichlund, O. J. Sordalen, O. Egeland, Control of vehicles with second-order nonholonomic constraints: underactuated vehicles, in: Proceedings of the European Control Conference, Rome, Italy, 1995, pp. 3086-3091.

[178] R. W. Brockett, Differential Geometric Control Theory, Boston, MA: Birkhauser, 1983, Ch. Asymptotic stability and feedback stabilization.

[179] W. N. White, J. Patenaude, M. Foss, D. Garcia, Direct Lyapunov approach for tracking control of underactuated mechanical systems, in: Proceedings of the American Control Conference, St. Louis, MO, USA, 2009, pp. 1341-1346.

[180] Z. Wang, Y. Guo, Unified control for Pendubot at four equilibrium points, IET Control Theory Appl 5 (2011) 155-163.

[181] G. He, Z. Geng, Robust backstepping control of an underactuated one-legged hopping robot in stance phase, Robotica 28 (2010) 583-596.

[182] H. Kazemi, V. J. Majd, M. M. Moghaddam, Modeling and robust backstepping control of an underactuated quadruped robot in bounding motion, Robotica (2012) $1-17$.

[183] H. Li, K. Furuta, F. L. Chernousko, Motion generation of the Capsubot using internal force and static friction, in: Proceedings of IEEE International Conference on Decision and Control, San Diego, CA, USA, 2006, pp. 6575-6580.

[184] Y. Liu, Analysis and control of underactuated mechanical systems, Ph.D. thesis, Faculty of Computing, Engineering and Technology, Staffordshire University (2010). 
[185] H. B. Fang, J. Xu, Dynamics of a mobile system with an internal accelerationcontrolled mass in a resistive medium, J. Sound and Vibration 330 (2011) 40024018.

[186] Y. Liu, M. Wiercigroch, E. Pavlovskaia, H. Yu, Modelling of a vibro-impact capsule system, Int J mechanical Sciences in press (2012) 1-13.

[187] R. Martinez, J. Alvarez, A controller for 2-DOF underactuated mechanical systems with discontinuous friction, Nonlinear Dynamics 53 (2008) 191-200.

[188] E. Garcia, A. Jimenez, P. Santos, M. Armada, The evolution of robotics research: from industrial robotics to field and service robotics, IEEE Robotics and Automation Magazine 14 (2007) 90-103.

[189] J. H. Shin, J. J. Lee, Fault detection and robust fault recovery control for robot manipulators with actuator failures, in: Proceedings of the IEEE International Conference on Robotics and Automation, Detroit, Michigan, USA, 1999, pp. 861-866.

[190] J. D. English, A. A. Maciejewski, Fault tolerance for kinematically redundant manipulators: Anticipating free-swinging joint failures, IEEE Trans Robotics and Automation 14 (1998) 566-575.

[191] A. A. G. Siqueira, M. H. Terra, Nonlinear and markovian $H_{\infty}$ controls of underactuated manipulators, IEEE Trans Control Systems Technology 12 (2004) 811-826.

[192] M. L. McIntyre, W. E. Dixon, D. M. Dawson, I. D. Walker, Fault identification for robot manipulators, IEEE Trans Robotics 21 (2005) 1028-1034.

[193] A. A. G. Siqueira, M. H. Terra, M. Bergerman, Robust Control of Robots: Fault Tolerant Approaches, Springer, 2011.

[194] T. Zilic, D. Pavkovic, D. Zorc, Modeling and control of a pneumatically actuated inverted pendulum, ISA Transactions 48 (2009) 327-335. 
[195] J. She, A. Zhang, X. Lai, M. Wu, Global stabilization of 2-DOF underactuated mechanical systems - an equivalent-input-disturbance approach, Nonlinear Dynamics 69 (2012) 495-509.

[196] C. Canudas, H. Olsson, K. J. Astrom, P. Lischinsky, A new model for control of systems with friction, IEEE Transaction on Automatic Control 40 (1995) 419-425.

[197] N. Barabanov, R. Ortega, Necessary and sufficient conditions for the passivity of the LuGre friction model, IEEE Transaction on Automatic Control 45 (2000) 830-832.

[198] C. Cornejo, L. Alvarez-Icaza, Passivity based control of underactuated mechanical systems with nonlinear dynamic friction, Journal of Vibration and Control 18 (2012) $1025-1042$.

[199] C. Chevallereau, Time-scaling control for an underactuated biped robot, IEEE Transactions on Robotics and Automation 19 (2003) 362-368.

[200] L. L. Flynn, R. Jafari, R. Mukherjee, Design and control of an underactuated threelink rolling biped, in: Proceedings of the IEEE International Conference on Robotics and Automation, Alaska, USA, 2010, pp. 3392-3397.

[201] C. Gosselin, F. Pelletier, T. Laliberte, An anthropomorphic underactuated robotic hand with 15 DOFs and a single actuator, in: Proceedings of the IEEE International Conference on Robotics and Automation, Pasadena, CA, USA, 2008, pp. 749-754.

[202] N. Miyashita, M. Kishikawa, M. Yamakita, 3d motion control of 2 links underactuated manipulator named AcroBOX, in: Proceedings of the American Control Conference, Minnesota, USA, 2006, pp. 5614-5619. 
(a)

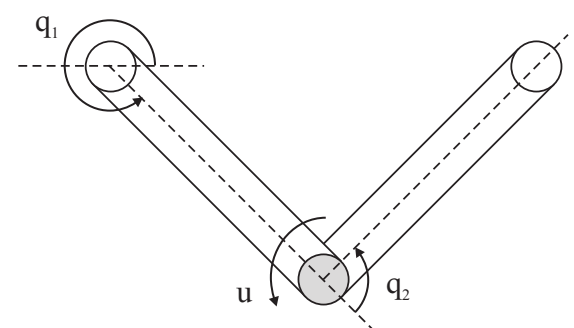

(b)

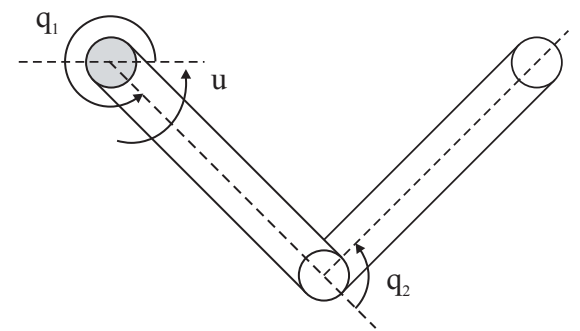

Figure 1: Two-link manipulators: (a) the Acrobot; (b) the Pendubot

(a)

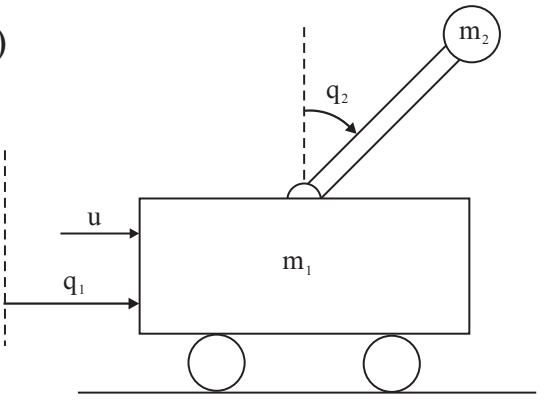

(b)

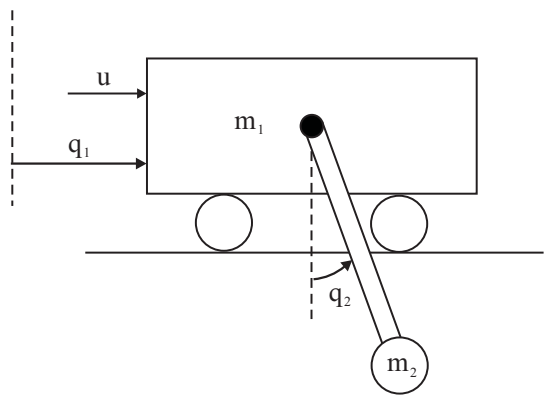

Figure 2: (a) The cart-pole system; (b) the crane system

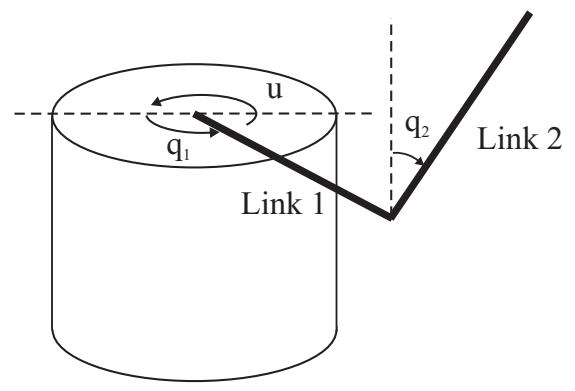

Figure 3: The rotating pendulum 


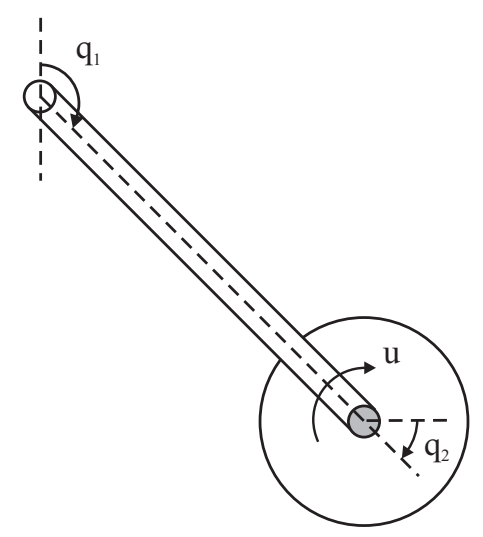

Figure 4: The IWP

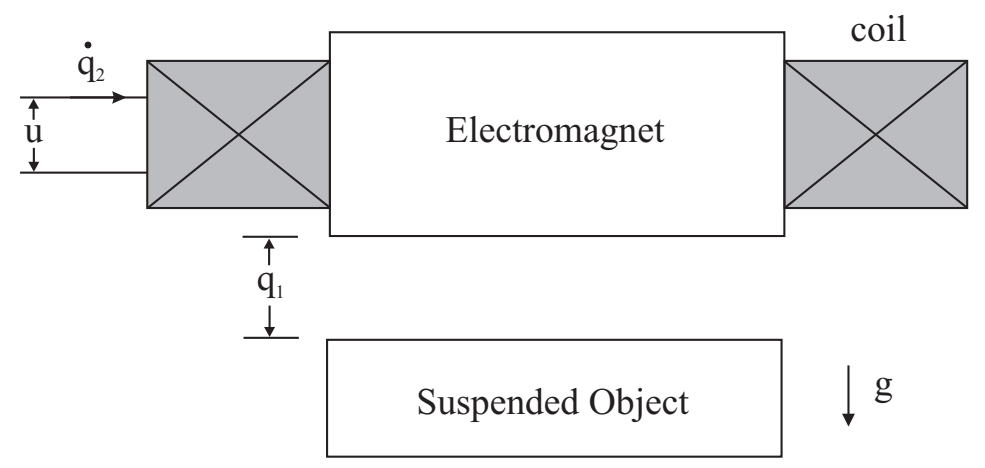

Figure 5: The magnetic suspension system

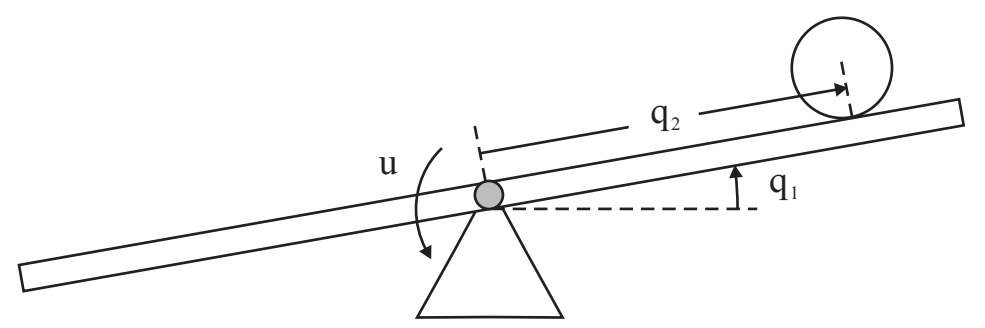

Figure 6: The ball-and-beam system

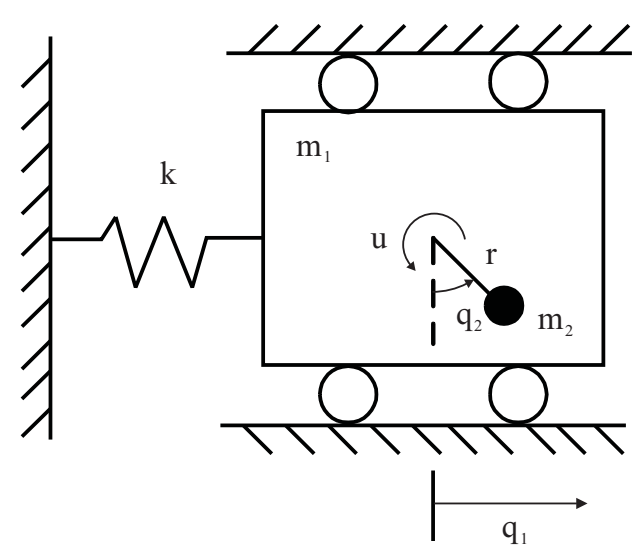

Figure 7: The TORA system 


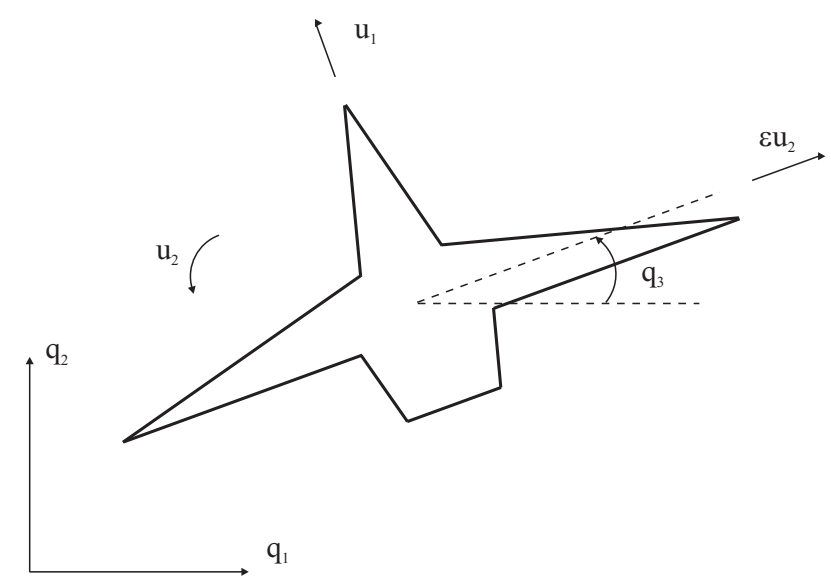

Figure 8: The VTOL aircraft

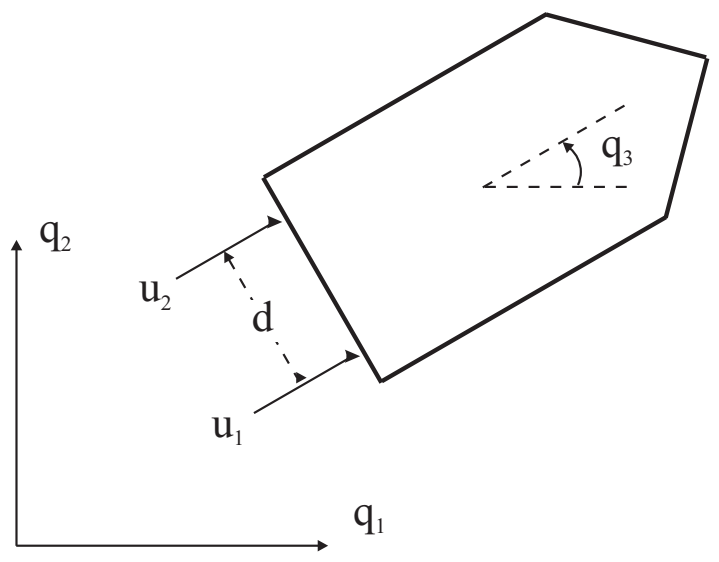

Figure 9: The surface vessel

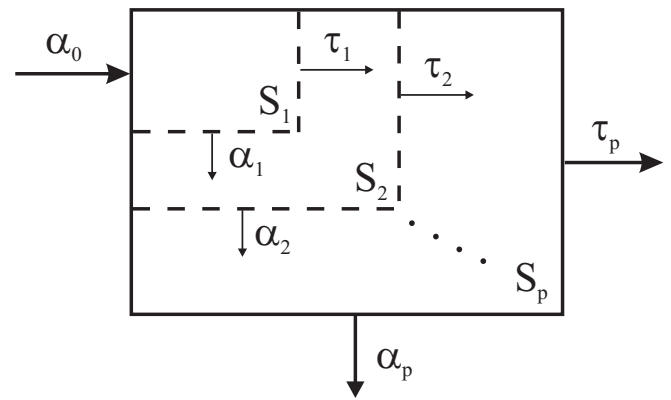

Figure 10: The schematics of backstepping 


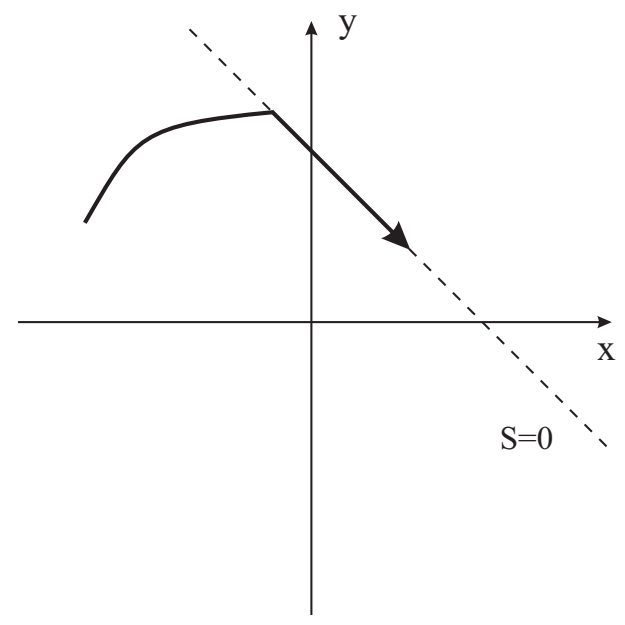

Figure 11: The schematics of SMC

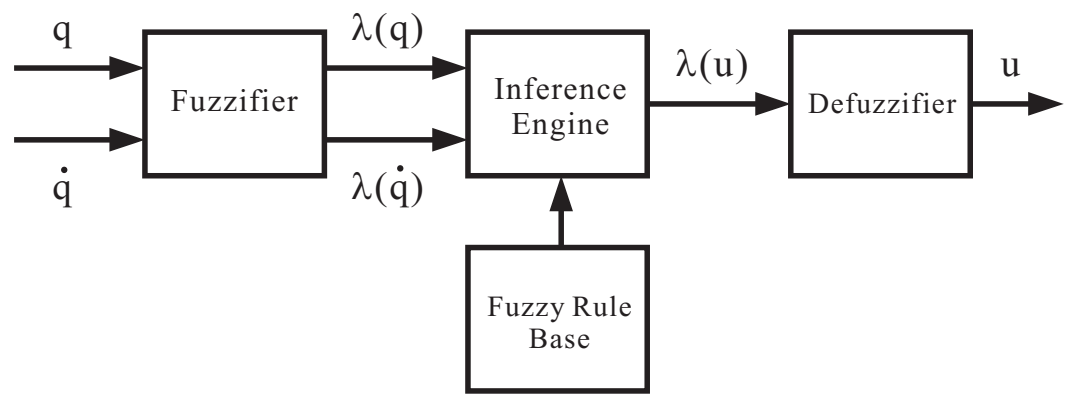

Figure 12: The schematics of FC

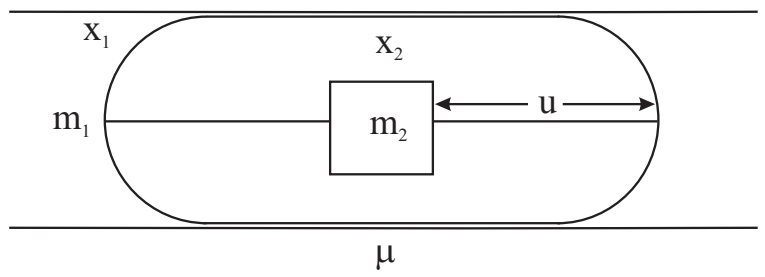

Figure 13: The Capsubot in a tube (adopted from [183]) 